\title{
Insignificant enhancement of export flux in the highly productive subtropical front, east of New Zealand: a high resolution study of particle export fluxes based on ${ }^{234} \mathrm{Th}:{ }^{238} \mathrm{U}$ disequilibria
}

\author{
K. Zhou ${ }^{1}$, S. D. Nodder ${ }^{2}$, M. Dai ${ }^{1}$, and J. A. Hall ${ }^{2}$ \\ ${ }^{1}$ State Key Lab of Marine Environmental Science, Xiamen University, Xiamen, China \\ ${ }^{2}$ National Institute of Water and Atmospheric Research Ltd. (NIWA), Private Bag 14-901, Wellington, New Zealand \\ Correspondence to: M. Dai (mdai@xmu.edu.cn)
}

Received: 10 September 2011 - Published in Biogeosciences Discuss.: 21 September 2011

Revised: 17 January 2012 - Accepted: 23 January 2012 - Published: 12 March 2012

\begin{abstract}
We evaluated the export fluxes of Particulate Organic Carbon (POC) in the Subtropical Frontal zone (STF) of the SW Pacific sector of the Southern Ocean. The site is characterized by enhanced primary productivity, which has been suggested to be stimulated through so-called natural iron fertilization processes where iron-depleted subantarctic water (SAW) mixes with mesotrophic, iron-replete subtropical water (STW). We adopted the small-volume ${ }^{234} \mathrm{Th}$ method to achieve the highest possible spatial sampling resolution in austral late autumn-early winter, MayJune, 2008. Inventories of chlorophyll- $a$, particulate ${ }^{234} \mathrm{Th}$ and POC observed in the upper $100 \mathrm{~m}$ were all elevated in the mid-salinity water type $(34.5<\mathrm{S}<34.8)$, compared with low salinity waters $(\mathrm{S}<34.5)$ which were of SAW origin with high macronutrients and high $(\mathrm{S}>34.8)$ salinity waters which were of STW origin with low macronutrients. However, Steady-State ${ }^{234} \mathrm{Th}$ fluxes were similar across the salinity gradient being, $25 \pm 0.78\left((1.5 \pm 0.047) \times 10^{3}\right)$ in the mid-salinity, and $29 \pm 0.53\left((1.8 \pm 0.032) \times 10^{3}\right)$ and $22 \pm 1.1 \mathrm{~Bq} \mathrm{~m}^{-2} \mathrm{~d}^{-1}\left((1.3 \pm 0.066) \times 10^{3} \mathrm{dpm} \mathrm{m}^{-2} \mathrm{~d}^{-1}\right)$ in the high and low salinity waters respectively. Bottle POC/Th ratios at the depth of $100 \mathrm{~m}$ were used to convert ${ }^{234} \mathrm{Th}$ fluxes into POC export fluxes. The derived POC flux did not appear to be enhanced in mid-salinity waters where the primary productivity was inferred to be the highest at the time of sampling, with a flux of $11 \pm 0.45 \mathrm{mmol} \mathrm{C} \mathrm{m}^{-2} \mathrm{~d}^{-1}$, compared to $14 \pm 0.39 \mathrm{mmol} \mathrm{C} \mathrm{m}^{-2} \mathrm{~d}^{-1}$ in high salinity waters and $8.5 \pm 0.66 \mathrm{mmol} \mathrm{C} \mathrm{m}^{-2} \mathrm{~d}^{-1}$ in low salinity waters. This study thus implied that natural iron fertilization does not necessarily lead to an enhancement of POC export in STF regions.
\end{abstract}

\section{Introduction}

The Subtropical Front (STF) (Fig. 1a) is a circum-global oceanographic feature, typically between about $35^{\circ} \mathrm{S}$ and $45^{\circ} \mathrm{S}$, where cold, high macro-nutrient, iron-limited subantarctic waters (SAW) mix with warm, low macro-nutrient, subtropical waters (STW) (Longhurst, 1998; Orsi et al., 1995). A number of studies have shown enhanced year-round chlorophyll concentrations and primary production (PP) in the STF region (Behrenfeld and Falkowski, 1997; Comiso et al., 1993; Murphy et al., 2001). The observed PP in the STF, east of New Zealand, can be as high as $22 \mathrm{mmol} \mathrm{C} \mathrm{m}^{-2} \mathrm{~d}^{-1}$ in winter, which may be more than 4 and 2-times higher than in the adjacent SAW and STW, respectively (BradfordGrieve et al., 1997). Similarly elevated integrated production rates have been observed in the STF around $152^{\circ} \mathrm{E}$ off Australia in summer (Clementson et al., 1998) and in the South African sector at $20^{\circ} \mathrm{E}$ in winter (Froneman et al., 1999). Such enhancement in PP in the STF zone has been suggested to be induced by natural iron fertilization processes, with the iron sourced from atmospheric deposition, shelf boundary exchange processes and/or mixing with iron-replete subtropical waters (Boyd et al., 1999, 2004; Pollard et al., 2009).

To the east of New Zealand, the STF is constrained bathymetrically along a prominent submarine ridge, the Chatham Rise (Heath, 1985; Uddstrom and Oien, 1999; Sutton, 2001). Like other STF zones, this site is the transition zone from mesotrophic STW in the North, characterized by the relatively high temperatures (summer $>18^{\circ} \mathrm{C}$; winter $\left.>14^{\circ} \mathrm{C}\right)$, salinities $(>35.1)$ and dissolved 


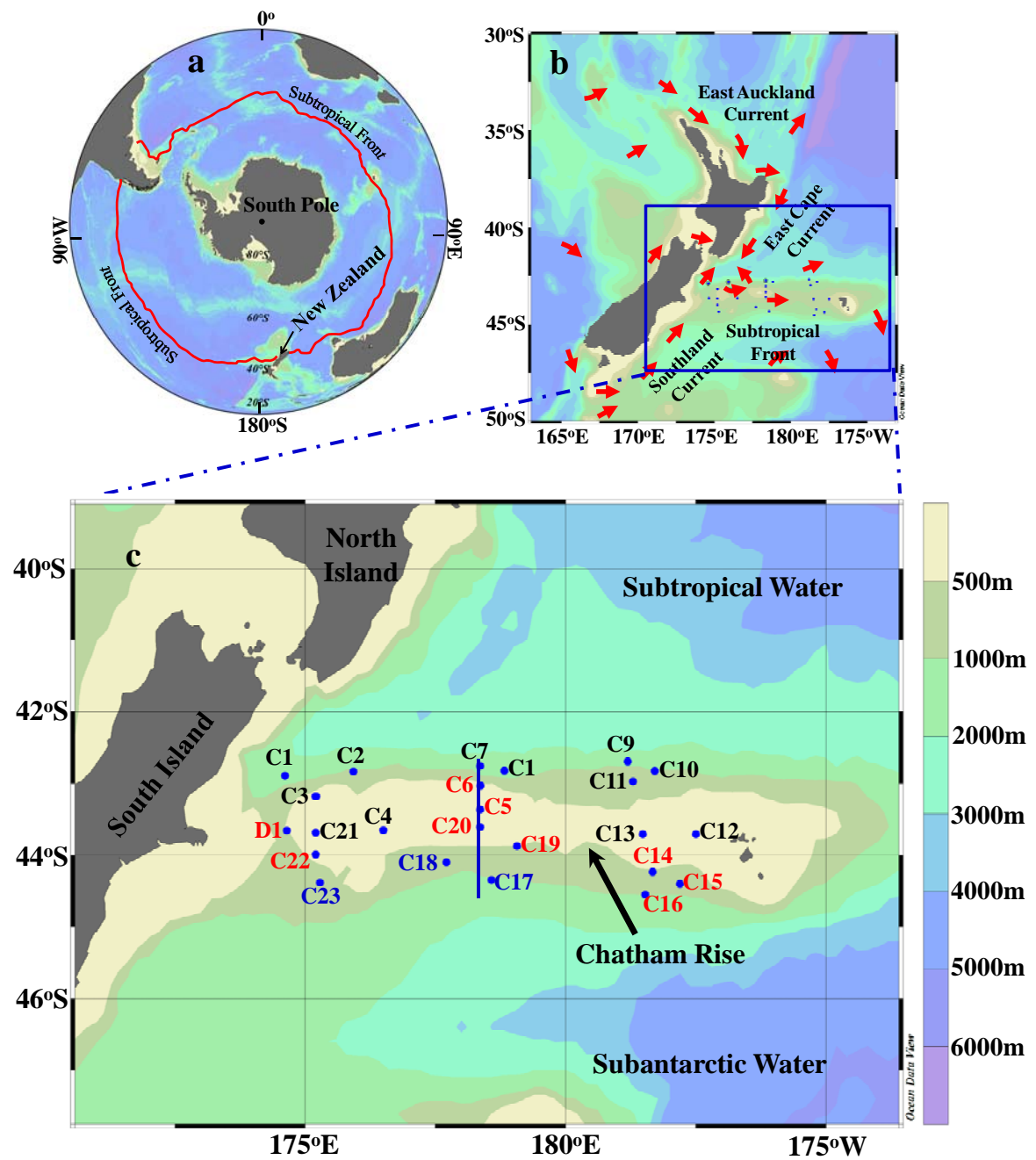

Fig. 1. Hemispherical map of the Southern Ocean showing the approximate location of the Subtropical Front as a red line (a), with the map of the study area showing the mean circulation and surface water masses (Nodder, 1997) (b); the location of sampling sites during the May-June 2008 research cruise TAN0806 (c); and the stations with salinities $>34.8$ (high), 34.5-34.8 (mid), and <34.5 (low) marked in black, red, and blue, respectively. The blue line represents the transect where stations were sampled throughout the water column (Transect M). The bathymetry of the study area is also shown to emphasize the location of the Chatham Rise.

iron levels $\left(>0.2 \mathrm{nmoll}^{-1}\right)$, and low macronutrient concentrations (e.g. $\mathrm{PO}_{4}<0.3 \mu \mathrm{mol} 1^{-1}$ ), to high nutrient-low chlorophyll (HNLC) SAW to the south, with typically low temperatures (summer $<14^{\circ} \mathrm{C}$; winter $<10^{\circ} \mathrm{C}$ ), low salinities $(<34.6)$ and low iron levels $\left(<0.1 \mathrm{nmol}^{-1}\right)$, but high macronutrient concentrations (e.g. $\mathrm{PO}_{4}>0.9 \mu \mathrm{moll}^{-1}$ ) (Boyd et al., 1999; Nodder, 1997). Regardless of seasonal and spatial variations, phytoplankton biomass and biological productivity are generally elevated over the Chatham Rise (Bradford-Grieve et al., 1997, 1999; Gall et al., 1999). In winter and spring, average chlorophyll $a$ (Chla) concentrations, integrated down to $100 \mathrm{~m}$, can reach up to $\sim 80 \mathrm{mg} \mathrm{m}^{-2}$, and the corresponding PP, integrated to $1 \%$ light level, can be more than $50 \mathrm{mmol} \mathrm{C} \mathrm{m}^{-2} \mathrm{~d}^{-1}$ (Bradford-Grieve et al., 1997). In comparison, the average inventory of Chl- $a$ and PP may be only $13 \mathrm{mg} \mathrm{m}^{-2}$ and $12 \mathrm{mmol} \mathrm{C} \mathrm{m}^{-2} \mathrm{~d}^{-1}$, respectively, in SAW and $33 \mathrm{mg} \mathrm{m}^{-2}$ and $46 \mathrm{mmol} \mathrm{C} \mathrm{m}^{-2} \mathrm{~d}^{-1}$ in STW. Such perennially high PP levels in the STF support a diverse planktonic and benthic ecosystem (Probert and McKnight, 1993; Bradford-Grieve et al., 1999) that seems to be translated to many fish species, with New Zealand's richest deep-water fisheries, primarily in blue grenadier (known locally as hoki, Macruronus novaezelandiae) and orange roughy (Hoplostethus atlanticus), occurring on the Chatham Rise (New Zealand Ministry of Fisheries, 2009).

However, little is known thus far about the spatio-temporal variability and magnitude of the downward export of particulate organic carbon (POC), or the fate of the PP in this frontal zone. Nodder (1997) used free-floating cylindrical sediment 
traps in the vicinity of the STF showing that the POC flux was less than $3 \mathrm{mmol} \mathrm{C} \mathrm{m}^{-2} \mathrm{~d}^{-1}$, which is in the same order of magnitude as that found in low export oligotrophic oceans. Indirect evidence from benthic studies, however, indicates that organic fluxes are enhanced on the crest and southern flank of the Chatham Rise (Probert and McKnight, 1993; Nodder et al., 2003). It is thus clear that information on the spatial variation of POC export within the STF is required if we are to evaluate whether the enhanced primary production in the frontal zone also leads to enhanced export production.

In the present study, we utilized a particle-reactive radionuclide, ${ }^{234} \mathrm{Th}\left(\mathrm{t}_{1 / 2}=24.1 \mathrm{~d}\right)$, as a tracer for particle export from the upper ocean. The technique has been applied widely in many oceanographic settings to examine processes occurring on time-scales of days to weeks (e.g. Coale and Bruland, 1985, 1987; Buesseler, 1992, 1998; Cochran and Masque, 2003; Dai and Benitez-Nelson, 2001; Waples et al., 2006). A further technological advantage is the use of the recently developed small-volume technique that enables high resolution sampling (Benitez-Nelson et al., 2001a; Buesseler et al., 2001a; Cai et al., 2006a). This method is essential in order to capture the particle dynamics and export flux variability in such regions as the STF, which are characterized by dynamic hydrography (e.g., Cai et al. 2008; Buesseler et al., 2009). Indeed, with high resolution sampling, Cai et al. (2008) observed significant variations of ${ }^{234} \mathrm{Th}$ deficit in the South China Sea, with enhanced Th and POC fluxes along the western and southern boundaries of this marginal sea. Buesseler et al. (2009) even observed high spatial variability in the northern subtropical gyre at ALOHA station off Hawaii, where a homogenous spatial distribution of POC flux from the surface ocean would have been expected.

The present study aims to examine the spatial distribution, magnitude and variability of upper ocean POC export in the STF over the Chatham Rise to the east of New Zealand by using high spatial resolution sampling of ${ }^{234} \mathrm{Th}$. We show that ${ }^{234} \mathrm{Th}$-based POC export is not significantly enhanced in the frontal zone, despite high Chl- $a$ and inferred PP levels, compared to the adjacent SAW and STW.

\section{Methods}

\subsection{Sample collection}

Samples were collected in late austral autumn-early winter from 23 May to 12 June in 2008 on board R/V Tangaroa, operated by the National Institute of Water and Atmospheric Research (NIWA) Ltd, New Zealand (NIWA voyage TAN0806). High spatial resolution sampling at 23 stations, covering a surface area of about $25000 \mathrm{~km}^{2}$, was conducted during the cruise (Fig. 1). Water samples were collected using 101 Niskin bottles mounted on a rosette sampler attached to a Seabird SBE9/11plus conductivity-temperature-depth
(CTD) sensor. A sub-sample of 41 seawater was used to determine the total ${ }^{234} \mathrm{Th}$ activity (see below) and another 81 was filtered onto a 25-mm diameter Quartz Microfiber (QMA, norminal pore size $\sim 1.0 \mu \mathrm{m}$ ) for particulate ${ }^{234} \mathrm{Th}$ and POC measurements. Samples were collected at 5 depths in the upper $100 \mathrm{~m}$ (normally 10, 20, 50, 70, and $100 \mathrm{~m}$ ), except along transect $\mathrm{M}$ where sampling was conducted at a finer depth resolution throughout the water column to better define the vertical structure of ${ }^{234} \mathrm{Th}$ over the Chatham Rise (shown as a blue line in Fig. 1). Since this was the first ${ }^{234}$ Th study over the Chatham Rise, such an intensive sampling strategy enabled a robust description of both the vertical and spatial distribution of ${ }^{234} \mathrm{Th}$.

\section{$2.2{ }^{234} \mathrm{Th}$ analysis}

We used the small-volume $\mathrm{MnO}_{2}$ co-precipitation technique for our total ${ }^{234} \mathrm{Th}$ analyses, as initially developed by Benitez-Nelson et al. (2001a) and Buesseler et al. (2001a), and further modified by Cai et al. (2006a). Four litre seawater samples were immediately acidified with $6 \mathrm{ml}$ concentrated $\mathrm{HNO}_{3}$ and spiked with $\sim 166.7 \mathrm{mBq}{ }^{230} \mathrm{Th}$ after collection. The samples were then mixed vigorously and allowed to stand for $12 \mathrm{~h}$ for isotopic equilibration. The $\mathrm{pH}$ was then brought up to 8.00-8.20 and thorium isotopes were co-precipitated with $\mathrm{MnO}_{2}$ by adding $0.25 \mathrm{ml} \mathrm{KMnO} 4$ (3.0 $\mathrm{gl}^{-1}$ ) and $0.25 \mathrm{ml} \mathrm{MnCl}_{2}\left(8.0 \mathrm{~g} \mathrm{MnCl}_{2} \cdot 4 \mathrm{H}_{2} \mathrm{Ol}^{-1}\right)$. The formation of the $\mathrm{MnO}_{2}$ precipitate was accelerated by heating to approximately $90^{\circ} \mathrm{C}$ in a water bath for $2 \mathrm{~h}$. The precipitate was then filtered onto a $25 \mathrm{~mm}$ QMA filter after samples were cooled to room temperature. The QMA filter was baked at about $100-200^{\circ} \mathrm{C}$ until dryness, and then mounted under a layer of Mylar and two layers of aluminum foil (total density $\sim 7.2 \mathrm{mg} \mathrm{m}^{-2}$ ) for beta-counting at sea by a gas-flow proportional low-level RIS $\varnothing$ beta-counter (Model GM-25-5, RISØ National Laboratory, Denmark). These samples were re-counted for background levels 6 months after the cruise (i.e., $>5$ half-lives of ${ }^{234} \mathrm{Th}$ ).

The QMA filter used for particulate ${ }^{234} \mathrm{Th}$ determination was dried at $50^{\circ} \mathrm{C}$ in an oven for $24 \mathrm{~h}$, and then mounted and beta-counted as above for the total ${ }^{234} \mathrm{Th}$. The average background levels for total and particulate ${ }^{234} \mathrm{Th}$ were 0.45 and 0.32 counts per minute (cpm), respectively.

We used an alpha spectrometric method for our total ${ }^{234} \mathrm{Th}$ recovery analysis (Cai et al., 2006a). Samples were demounted after background counting, spiked with $\sim 166.7 \mathrm{mBq}{ }^{228} \mathrm{Th}\left({ }^{232} \mathrm{U}_{-}{ }^{228} \mathrm{Th}\right.$ solution $)$ and then digested by adding $10 \mathrm{ml}$ concentrated $\mathrm{HNO}_{3}, 1 \mathrm{ml} \mathrm{HF}$ and $1 \mathrm{ml} \mathrm{H}_{2} \mathrm{O}_{2}$. Thorium isotopes were purified through iron precipitation and a classic anion column. Eluents were then evaporated onto a $25 \mathrm{~mm}$ stainless steel disc after extraction using $1.5 \mathrm{ml}$ of $0.25 \mathrm{moll}^{-1}$ theonyl trifluoroacetone(TTA)/benzene solution. The disc was counted by alpha spectrometry (Octete ${ }^{\mathrm{TM}} \mathrm{PC}$ ) until both thorium isotopes $\left({ }^{230} \mathrm{Th}\right.$ and $\left.{ }^{228} \mathrm{Th}\right)$ reached more than 2,500 counts. Most of 
the final recoveries for ${ }^{230} \mathrm{Th}$ were between $80 \%$ and $103 \%$. The average of the recoveries was $90.2 \pm 1.4 \%$ (mean \pm 1 standard deviation). The errors associated with ${ }^{234}$ Th activity determination are propagated from the counting errors on the first counts, background measurements and recovery analyses. The precision of the final ${ }^{234} \mathrm{Th}$ activity was better than $5 \%$.

Instead of undertaking specific sample analyses, we used the relationship: $\mathrm{A}_{\mathrm{U}}\left(\mathrm{mBq}^{-1}\right)=1.1801 \times$ salinity to estimate the ${ }^{238} \mathrm{U}$ activity $\left(\mathrm{A}_{\mathrm{U}}\right)$ in the seawater according to Chen et al. (1986). The uncertainty of ${ }^{238} \mathrm{U}$ activity was $\sim 3 \%$ which was also included in the error estimates associated with the ${ }^{234} \mathrm{Th}$ fluxes.

\subsection{POC and Particulate Nitrogen (PN) analysis}

After beta-counting for particulate ${ }^{234} \mathrm{Th}$, the samples were demounted for POC and PN analysis. The filters were placed in Petri dishes and fumed using concentrated hydrochloric acid for $24 \mathrm{~h}$ to remove the carbonate phase. POC concentrations were then determined by a PE-2400 SERIES II CHNS/O analyzer, according to JGOFS protocols (Knap et al., 1996). Replicate procedural C blanks from sampling to instrumental carbon determination have been tested before (Chen, 2008), and were all less than $6 \mu \mathrm{g} \mathrm{C}$ and $2 \mu \mathrm{g} \mathrm{N}$, which typically accounted for less than $10 \%$ of the sample POC and $\mathrm{PN}$, respectively. The precision for our POC measurements were always better than $10 \%$ (Cai et al., 2006a; Chen, 2008).

\subsection{Other ancillary parameters}

The temperature and salinity vertical profile data were obtained from the Seabird SBE9/11plus CTD. A Wetlabs fluorometer, interfaced with the CTD, was used to determine the fluorescence at an excitation and emission wavelength of 470 and $695 \mathrm{~nm}$. In order to calibrate the fluorometer, discrete seawater samples (1-21) were collected for the measurement of Chl- $a$. Briefly, seawater was filtered through a Whatman GF/F filter, and Chl- $a$ on the filter was then extracted with $90 \%$ acetone and analyzed using a spectrofluorometer. A linear relationship was found between fluorescence and Chl- $a$ $\left(\right.$ Chl $-a\left(\mu \mathrm{g} \mathrm{l}^{-1}\right)=0.639 \times$ Fluorescence, $\left.R^{2}=0.87, n=50\right)$, which was then applied to convert the fluorescence values into Chl- $a$ concentrations. Uncertainties for the Chl- $a$ determination were $<10 \%$.

Macronutrients were determined using classic colorimetric method (Ellwood and Maher, 2003). The detection limits for dissolved inorganic nitrogen (DIN, nitrate plus nitrite), $\mathrm{PO}_{4}$ and $\mathrm{Si}(\mathrm{OH})_{4}$ were $0.07 \mu \mathrm{mol} 1^{-1}, 0.03 \mu \mathrm{moll}^{-1}$ and $0.07 \mu \mathrm{moll}^{-1}$, respectively.

\section{Results}

\subsection{Hydrography}

The complex hydrological characteristics of the STF are shown by the relationship between potential temperature and salinity in Fig. 2. In the upper ocean, distinctly different water characteristics were found between stations, representing different degrees of mixing. The surface temperature and salinity changed dramatically over the sampled area from lows of $9.4^{\circ} \mathrm{C}$ and 34.2 , respectively, in surface waters with SAW affinities, to highs of $15.2^{\circ} \mathrm{C}$ and 35.2 in surface waters associated with STW.

Figure 3 shows the spatial distributions of temperature and salinity at $2 \mathrm{~m}$ and $100 \mathrm{~m}$ water depths. The gradients of salinity and temperature were much greater in the region between $43^{\circ} \mathrm{S}$ and $44^{\circ} \mathrm{S}$, coinciding with the crest and upper southern flank of the Chatham Rise. Vertically, the surface $150 \mathrm{~m}$ of all stations sampled in the STF was well stratified (Fig. 4a, b, and c). However, the depth of the surface isothermal mixed-layer, defined by the density surface with a $0.5^{\circ} \mathrm{C}$ temperature difference from the reference depth (Kara et al., 2000), was not uniform between stations, ranging from $54 \mathrm{~m}$ to $178 \mathrm{~m}$. Typically and as illustrated in Fig. 4, the depth of the mixed-layer at high salinity stations was deeper than at mid- and low salinity stations (see next section for definitions of water types).

\subsection{Chl- $a$, macronutrient, and POC distribution}

Vertically, Chl- $a$ was highest within the upper mixed-layer. Below the mixed-layer, the Chl- $a$ concentration decreased to background, effectively zero (Fig. 4a, b). Regionally, Chl- $a$ concentrations were elevated where salinities were between 34.5 and 34.8 (Fig. 2b). In such cases, the average Chl- $a$ concentration in the upper $100 \mathrm{~m}$ was as high as $0.79 \mathrm{\mu g}^{-1} \mathrm{com}$ pared to $0.40 \mu \mathrm{g} 1^{-1}$ at salinities $>34.8$ or $0.37 \mu \mathrm{g} 1^{-1}$ at salinities $<34.5$. Accordingly, for the ease of discussion, we have divided the STF waters of our study area into three different water types: low $(\mathrm{S}<34.8)$, mid- $(34.5<\mathrm{S}<34.8)$ and high salinity ( $\mathrm{S}>34.8$ ), according to the vertical and horizontal distribution of Chl- $a$.

Both DIN and $\mathrm{PO}_{4}$ concentrations were generally at replete levels during the cruise. In the upper $100 \mathrm{~m}$, DIN concentrations ranged from $3.6 \mu \mathrm{moll}^{-1}$ to $13.9 \mu \mathrm{mol}^{-1}$ in mid-salinity waters, with an average of $8.9 \mu \mathrm{moll}^{-1}$. In high salinity waters, it was lowest, ranging from $1.1 \mu \mathrm{mol}^{-1}$ to $12.3 \mu \mathrm{moll}^{-1}$, with an average of $4.7 \mu \mathrm{moll}^{-1}$. In low salinity waters, DIN concentration varied from $7.8 \mu \mathrm{moll}^{-1}$ to $14.9 \mu \mathrm{mol}^{-1}$, with an average of $11.3 \mu \mathrm{mol}^{-1}$, which was the highest among the three water types. $\mathrm{PO}_{4}$ shared the similar distribution pattern with that of DIN. In mid salinity waters, the average of $\mathrm{PO}_{4}$ concentration in the upper $100 \mathrm{~m}$ was $0.71 \mu \mathrm{mol}^{-1}$, compared to $0.42 \mu \mathrm{mol}^{-1}$ in high salinity waters and $0.87 \mu \mathrm{moll}^{-1}$ in low salinity waters. 

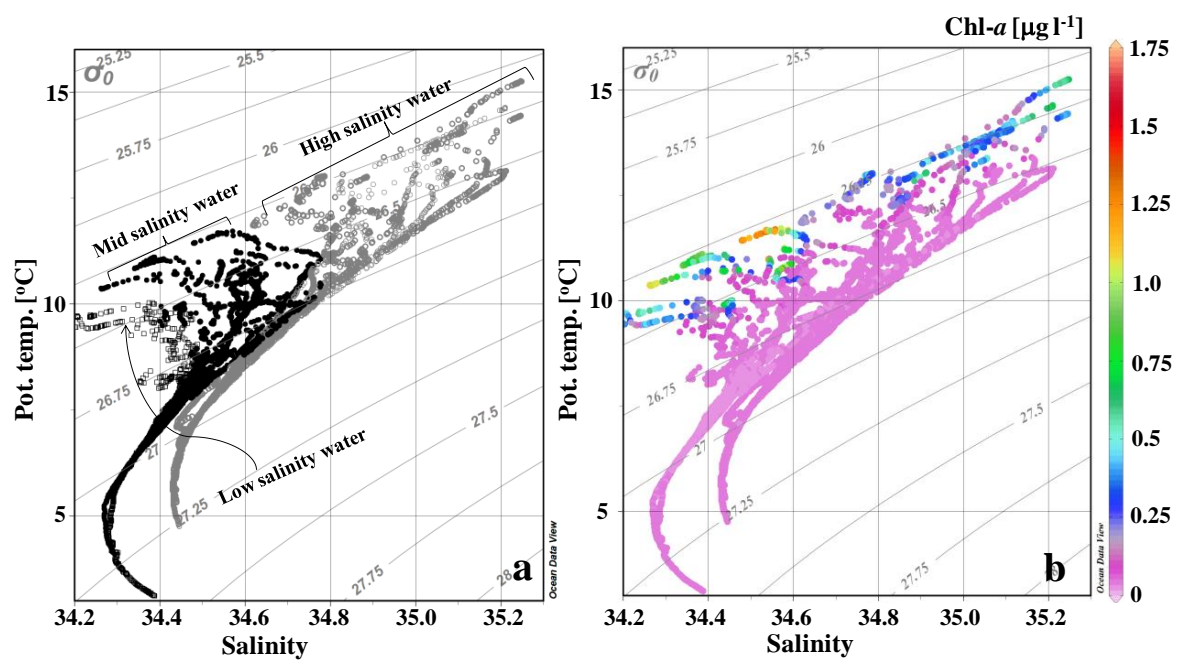

Fig. 2. (a) T-S diagram over the Chatham Rise, east of New Zealand (o high salinity ( $\mathrm{S}>34.8$ ) water; $\bullet$ mid-salinity $(34.5<\mathrm{S}<34.8)$ water; $\square$ low salinity $(\mathrm{S}<34.5)$ waters). The isopycnal lines are also shown; (b) Regional distribution of Chl- $a$ plotted on the T-S diagram.
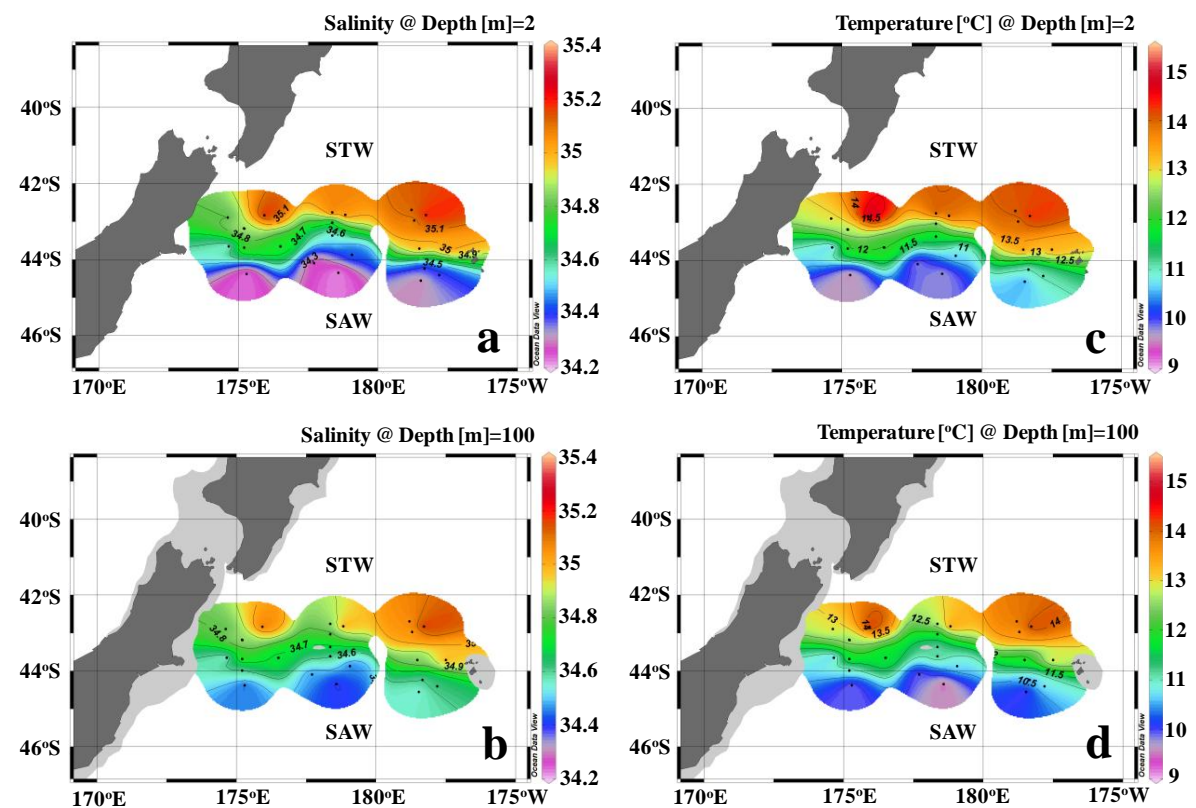

Fig. 3. Distributions of temperature and salinity, highlighting dramatic changes within the Subtropical Frontal zone: (a) surface salinity at $2 \mathrm{~m}$ water depth, (b) salinity at $100 \mathrm{~m}$, (c) surface temperature, and (d) temperature at $100 \mathrm{~m}$. The Subtropical water (STW) and Subantarctic water (SAW) are also highlighted.

$\mathrm{Si}(\mathrm{OH})_{4}$ was only measured at stations $\mathrm{C} 1$ through $\mathrm{C} 7$ covering high and mid-salinity waters. Based on the limited $\mathrm{Si}(\mathrm{OH})_{4}$ data set, its concentration in the upper $100 \mathrm{~m}$ varied from 0.39 to $3.43 \mu \mathrm{mol}^{-1}$ in mid-salinity stations (C5 and C6), which is comparable to the range in the high salinity stations $\left(\mathrm{C} 1, \mathrm{C} 2, \mathrm{C} 3, \mathrm{C} 4\right.$ and $\left.\mathrm{C} 7,0.91-3.11 \mu \mathrm{moll}^{-1}\right)$. Inventories of these macronutrients are listed in Table 2.
POC concentrations varied from $0.40 \mu \mathrm{molCl}^{-1}$ to $6.1 \mu \mathrm{mol} \mathrm{Cl} l^{-1}$ across the study area (Table 1$)$. In the upper mixed-layer, POC distributions generally followed that of Chl- $a$, indicating a relationship between POC and phytoplankton biomass. Regionally, POC concentration was also relatively enhanced in mid-salinity waters. The average POC concentration in the upper $100 \mathrm{~m}$ was $3.9 \mu \mathrm{mol} \mathrm{Cl}^{-1}$ in this water type, compared to $2.4 \mu \mathrm{mol} \mathrm{Cl}{ }^{-1}$ and $2.6 \mu \mathrm{mol} \mathrm{Cl} l^{-1}$ in high and low salinity waters, respectively. In a departure 


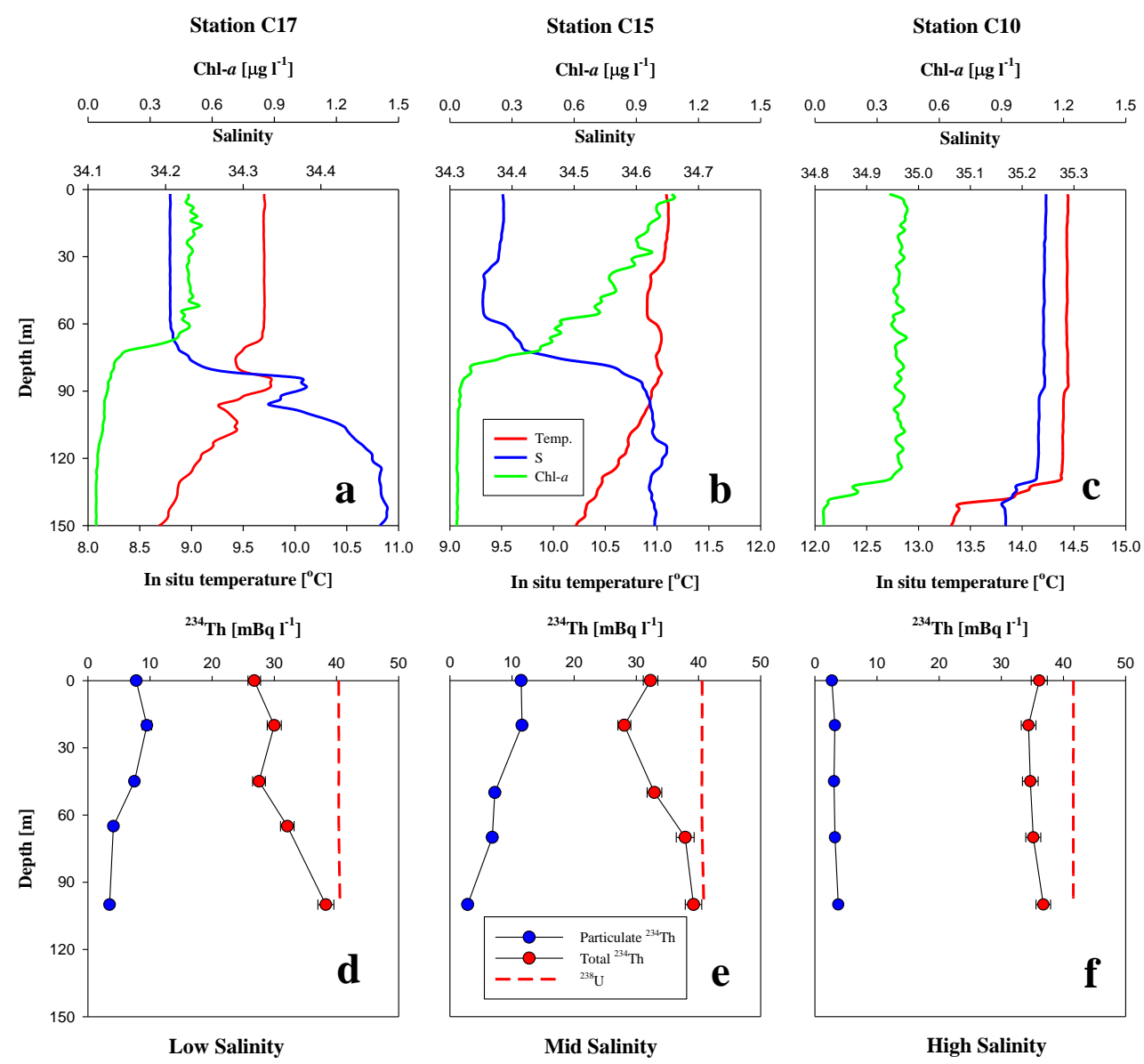

Fig. 4. (a), (b), and (c) Vertical profiles of temperature, salinity and Chl- $a$ in the upper $100 \mathrm{~m}$ at stations $\mathrm{C} 17, \mathrm{C} 15$, and C10, respectively. (c), (d), and (e) Vertical profiles of particulate and total ${ }^{234} \mathrm{Th}$ from the same stations.
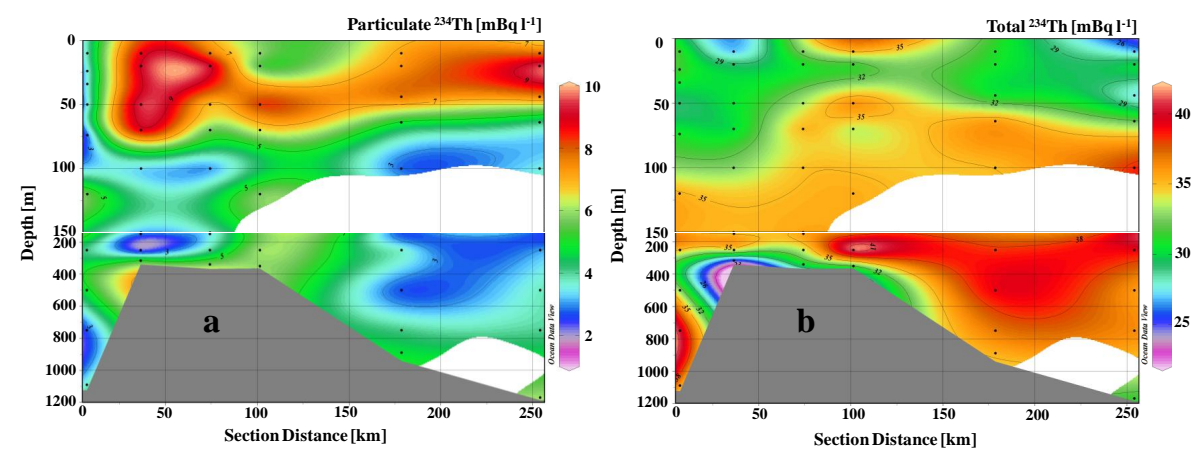

Fig. 5. Sectional distributions of ${ }^{234}$ Th activity along a meridional transect (Transect M) across the Chatham Rise (see Fig. 1 for transect location): (a) particulate ${ }^{234} \mathrm{Th}$ and (b) total ${ }^{234} \mathrm{Th}$.

from the vertical distribution of Chl- $a$, POC concentrations were often higher near the bottom of the water column, which may be indicative of near-bottom sediment resuspension (e.g., Nodder, 1997; Nodder et al., 2007).

The $\mathrm{C} / \mathrm{N}$ ratio was quite stable in the upper ocean, ranging from 5.2 to 8.5 , with an average of $6.6 \pm 1.5(n=146)$ (mean \pm 1 standard deviation), which is almost identical to the Redfield ratio of 6.63 (Redfield et al., 1963). No obvious changes in $\mathrm{C} / \mathrm{N}$ ratio were found in association with changes in salinity, suggesting that the particles in the study area were predominantly biogenic in origin. 

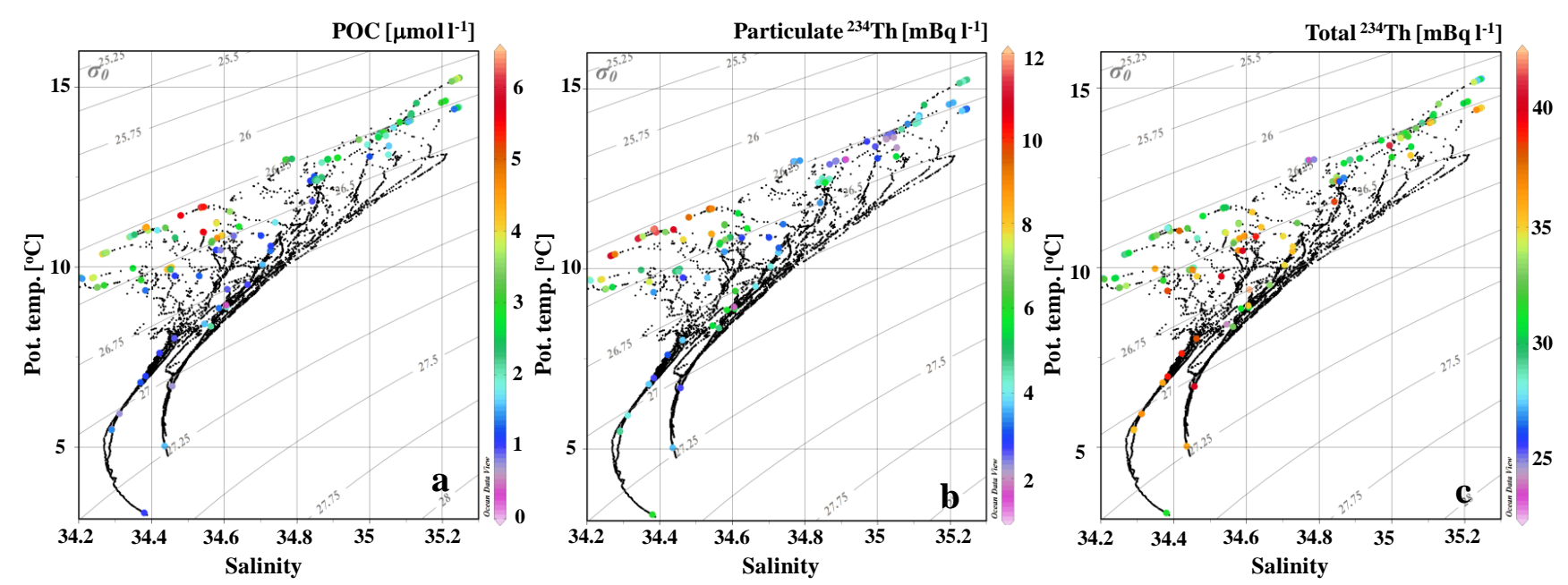

Fig. 6. Regional distributions of: (a) particulate organic carbon, (b) particulate ${ }^{234} \mathrm{Th}$, and (c) total ${ }^{234} \mathrm{Th}$ plotted on a T-S diagram.

\section{3 $\quad{ }^{234}$ Th distribution}

\subsubsection{Vertical profiles of ${ }^{234} \mathrm{Th}$}

Particulate and total ${ }^{234} \mathrm{Th}$ activities are listed in Table 1. Profiles of three representative stations from low, mid- and high salinity waters are shown in Fig. 4. The activities of total ${ }^{234} \mathrm{Th}$ varied from $24 \pm 1.0 \mathrm{mBq}^{-1}$ to $42 \pm 2.4 \mathrm{mBq}^{-1}$. Particulate ${ }^{234} \mathrm{Th}$ activities on suspended particles were variable, ranging from $1.7 \pm 0.15 \mathrm{mBq}^{-1}$ to $12 \pm 0.29 \mathrm{mBq}^{-1}$. The ${ }^{234} \mathrm{Th}$ activity profile in the upper $100 \mathrm{~m}$ was related to the vertical distribution of Chl- $a$. As illustrated in Fig. 4, total ${ }^{234} \mathrm{Th}$ activity was lowest within the isothermal mixed layer. Below the mixed-layer, the activity of total ${ }^{234} \mathrm{Th}$ began to increase as the Chl- $a$ leveled off, until it reached secular equilibrium with ${ }^{238} \mathrm{U}$ at the base of the euphotic zone (Ez) where Chl- $a$ reached its minimum. At stations with depths of Ez greater than $100 \mathrm{~m}$, the total ${ }^{234} \mathrm{Th}$ activity was in deficit with respect to ${ }^{238} \mathrm{U}$ from $0-100 \mathrm{~m}$. The vertical distribution of particulate ${ }^{234} \mathrm{Th}$ also generally resembled that of Chl- $a$ and POC, in that its activity was homogenous within and decreased below the mixed-layer. Not surprisingly, higher particulate ${ }^{234} \mathrm{Th}$ activities were related to higher levels of Chl- $a$, indicating intensive scavenging of biogenic particles in high Chl- $a$ waters. However, total ${ }^{234} \mathrm{Th}$ activities seemed to be independent of Chl- $a$ and/or biomass. As shown in Fig. 4, for example, the Chl- $a$ concentration at the mid-salinity station $\mathrm{C} 15$ was twice at the low salinity station $\mathrm{C} 17$, but the activities of total ${ }^{234} \mathrm{Th}$ were similar at each station.

\subsubsection{Meridional distribution of ${ }^{234} \mathrm{Th}$}

To better describe the spatial distribution pattern of ${ }^{234} \mathrm{Th}$ in the STF across the Chatham Rise, transect M, consisting of stations C5, C6, C7, C17, C18, and C20, was sampled throughout the water column (shown in Fig. 5). Generally, total ${ }^{234} \mathrm{Th}$ was in deficit in upper and near-bottom waters, and was in equilibrium with ${ }^{238} \mathrm{U}$ over the mid-water column. No meridional trends were found in the upper water column along transect M. Particulate ${ }^{234} \mathrm{Th}$ was higher within the Ez and in bottom waters than in the mid-water column. Interestingly, at mid-depths of 300-600 m, a deficit of total ${ }^{234} \mathrm{Th}$ was found in waters on both sides of the Chatham Rise crest. This observation may reflect the horizontal transport of the re-suspended particles along the Chatham Rise flanks, consistent with the near-bottom increases in particle fluxes from sediment trap (Nodder, 1997; Nodder and Northcote, 2001) and benthic studies (Nodder et al., 2007) in the area.

\subsubsection{Regional distribution of ${ }^{234} \mathrm{Th}$}

To provide a composite view of the regional distribution of ${ }^{234} \mathrm{Th}$ in relation to different water types, the total and particulate ${ }^{234} \mathrm{Th}$ activities are superimposed on a T/S diagram (Fig. 6). Similar to Chl- $a$ and POC, the activities of particulate ${ }^{234} \mathrm{Th}$ in the upper ocean were enhanced in mid-salinity waters, where the average of particulate ${ }^{234} \mathrm{Th}$ activities increased up to $7.5 \pm 0.063 \mathrm{mBq}^{-1}$, which was two times higher than in the high salinity waters $\left(3.3 \pm 0.035 \mathrm{mBq}^{-1}\right)$. Noticeably, however, high activities of particulate ${ }^{234} \mathrm{Th}$ were not necessarily correlated with low total ${ }^{234} \mathrm{Th}$ activities. Such a distribution pattern also held for total ${ }^{234} \mathrm{Th}$ among the three water types. The activities of total ${ }^{234} \mathrm{Th}$ in mid-salinity waters varied from $24 \pm 3.0 \mathrm{mBq}^{-1}$ to $42 \pm 2.4 \mathrm{mBq}^{-1}$, with an average of $33 \pm 0.23 \mathrm{mBql}^{-1}$, and similarly from $23 \pm 0.94 \mathrm{mBq}^{-1}$ to $41 \pm 1.3 \mathrm{mBq}^{-1}$ in the high salinity waters, with an average of $32 \pm 0.16 \mathrm{mBq}^{-1}$. In comparison, total ${ }^{234} \mathrm{Th}$ activity ranged from $27 \pm 1.0$ to $39 \pm 1.3 \mathrm{mBql}^{-1}$ in low salinity waters, with an average of $34 \pm 0.32 \mathrm{mBq}^{-1}$. 
Table 1. Temperature, Salinity, Particulate and Total ${ }^{234} \mathrm{Th}$ activities, ${ }^{238} \mathrm{U}$ activities, ${ }^{234} \mathrm{Th}:{ }^{238} \mathrm{U}$ and POC: ${ }^{234} \mathrm{Th}$ ratios for all stations in the Subtropical Front, Chatham Rise, New Zealand, measured in May-June 2008 (NIWA cruise TAN0806).

\begin{tabular}{|c|c|c|c|c|c|c|c|c|}
\hline $\begin{array}{r}\text { Depth } \\
\mathrm{m}\end{array}$ & $\begin{array}{r}\text { Temp. } \\
{ }^{\circ} \mathrm{C}\end{array}$ & Salinity & $\begin{array}{r}\text { Particulate }{ }^{234} \mathrm{Th} \\
\mathrm{mBql}^{-1}\end{array}$ & $\begin{array}{c}\text { Total }^{234} \mathrm{Th} \\
\mathrm{mBq}^{-1}\end{array}$ & $\begin{array}{r}{ }^{238} \mathrm{U} \\
\mathrm{mBq} 1^{-1}\end{array}$ & $\begin{array}{r}\mathrm{POC} \\
\mu \mathrm{mol} \mathrm{C} 1^{-1}\end{array}$ & ${ }^{234} \mathrm{Th}:{ }^{238} \mathrm{U}$ & $\begin{array}{r}\mathrm{C}: \text { Th Ratio } \\
\mathrm{mmol} \mathrm{C} \mathrm{Bq}^{-1}\end{array}$ \\
\hline \multicolumn{9}{|c|}{ High Salinity Stations } \\
\hline \multicolumn{9}{|c|}{$\mathrm{C} 1,174^{\circ} 36^{\prime} \mathrm{E}, 42^{\circ} 53^{\prime} \mathrm{S}, 1010 \mathrm{~m}, 05-24-2008$} \\
\hline 10 & 12.979 & 34.769 & $2.8 \pm 0.18$ & $24 \pm 1.5$ & $41 \pm 1.2$ & $2.9 \pm 0.29$ & $0.58 \pm 0.041$ & $1.0 \pm 0.12$ \\
\hline 20 & 12.980 & 34.769 & $2.4 \pm 0.20$ & $28 \pm 1.1$ & $41 \pm 1.2$ & $2.9 \pm 0.29$ & $0.69 \pm 0.033$ & $1.2 \pm 0.16$ \\
\hline 50 & 12.989 & 34.771 & $2.5 \pm 0.17$ & $29 \pm 1.1$ & $41 \pm 1.2$ & $2.7 \pm 0.27$ & $0.70 \pm 0.034$ & $1.1 \pm 0.13$ \\
\hline 70 & 12.992 & 34.772 & $3.7 \pm 0.18$ & $23 \pm 0.94$ & $41 \pm 1.2$ & $3.1 \pm 0.31$ & $0.57 \pm 0.029$ & $0.86 \pm 0.10$ \\
\hline 100 & 13.020 & 34.786 & $3.4 \pm 0.21$ & $25 \pm 0.99$ & $41 \pm 1.2$ & $2.4 \pm 0.24$ & $0.61 \pm 0.030$ & $0.70 \pm 0.081$ \\
\hline \multicolumn{9}{|c|}{$\mathrm{C} 2,175^{\circ} 56^{\prime} \mathrm{E}, 42^{\circ} 50^{\prime} \mathrm{S}, 687 \mathrm{~m}, 05-25-2008$} \\
\hline 10 & 15.256 & 35.247 & $4.7 \pm 0.21$ & $27 \pm 1.4$ & $42 \pm 1.3$ & $3.3 \pm 0.33$ & $0.66 \pm 0.040$ & $0.70 \pm 0.077$ \\
\hline 20 & 15.234 & 35.242 & $4.8 \pm 0.23$ & $31 \pm 1.1$ & $42 \pm 1.3$ & $3.8 \pm 0.38$ & $0.74 \pm 0.034$ & $0.80 \pm 0.089$ \\
\hline 50 & 15.207 & 35.235 & $4.3 \pm 0.20$ & $27 \pm 1.1$ & $42 \pm 1.3$ & $3.7 \pm 0.37$ & $0.66 \pm 0.033$ & $0.86 \pm 0.095$ \\
\hline 70 & 15.168 & 35.226 & $4.7 \pm 0.21$ & $34 \pm 1.6$ & $42 \pm 1.3$ & $3.4 \pm 0.34$ & $0.82 \pm 0.045$ & $0.73 \pm 0.080$ \\
\hline 100 & 14.564 & 35.129 & $5.1 \pm 0.23$ & $31 \pm 1.1$ & $42 \pm 1.2$ & $2.4 \pm 0.24$ & $0.76 \pm 0.035$ & $0.46 \pm 0.051$ \\
\hline \multicolumn{9}{|c|}{$\mathrm{C} 3,175^{\circ} 13^{\prime} \mathrm{E}, 43^{\circ} 11^{\prime} \mathrm{S}, 125 \mathrm{~m}, 05-26-2008$} \\
\hline 10 & 12.983 & 34.885 & $2.5 \pm 0.19$ & $27 \pm 1.3$ & $41 \pm 1.2$ & $2.8 \pm 0.28$ & $0.66 \pm 0.036$ & $1.1 \pm 0.14$ \\
\hline 20 & 12.990 & 34.884 & $2.6 \pm 0.20$ & $32 \pm 1.1$ & $41 \pm 1.2$ & $2.8 \pm 0.28$ & $0.78 \pm 0.036$ & $1.1 \pm 0.14$ \\
\hline 50 & 13.002 & 34.886 & $2.5 \pm 0.18$ & $24 \pm 1.1$ & $41 \pm 1.2$ & $2.8 \pm 0.28$ & $0.59 \pm 0.031$ & $1.2 \pm 0.14$ \\
\hline 70 & 12.998 & 34.884 & $2.4 \pm 0.17$ & $29 \pm 1.0$ & $41 \pm 1.2$ & $2.5 \pm 0.25$ & $0.71 \pm 0.033$ & $1.1 \pm 0.13$ \\
\hline 100 & 12.917 & 34.868 & $2.4 \pm 0.20$ & $31 \pm 1.1$ & $41 \pm 1.2$ & $2.0 \pm 0.20$ & $0.75 \pm 0.034$ & $0.82 \pm 0.11$ \\
\hline \multicolumn{9}{|c|}{$C 3-2^{\mathrm{a}}, 06-11-2008$} \\
\hline 10 & 12.487 & 34.867 & $4.2 \pm 0.62$ & $27 \pm 1.8$ & $41 \pm 1.2$ & $2.4 \pm 0.24$ & $0.66 \pm 0.049$ & $0.56 \pm 0.10$ \\
\hline 20 & 12.490 & 34.867 & $3.3 \pm 0.68$ & $25 \pm 2.3$ & $41 \pm 1.2$ & $2.0 \pm 0.20$ & $0.61 \pm 0.059$ & $0.61 \pm 0.14$ \\
\hline 50 & 12.490 & 34.867 & $4.2 \pm 0.60$ & $25 \pm 1.8$ & $41 \pm 1.2$ & $2.1 \pm 0.21$ & $0.61 \pm 0.047$ & $0.48 \pm 0.084$ \\
\hline 70 & 12.498 & 34.868 & $4.4 \pm 0.60$ & $27 \pm 1.7$ & $41 \pm 1.2$ & $2.3 \pm 0.23$ & $0.65 \pm 0.046$ & $0.52 \pm 0.087$ \\
\hline 100 & 12.404 & 34.856 & $5.6 \pm 0.68$ & $26 \pm 1.8$ & $41 \pm 1.2$ & $2.1 \pm 0.21$ & $0.64 \pm 0.049$ & $0.38 \pm 0.060$ \\
\hline \multicolumn{9}{|c|}{$\mathrm{C} 4,176^{\circ} 03^{\prime} \mathrm{E}, 43^{\circ} 29^{\prime} \mathrm{S}, 387 \mathrm{~m}, 05-26-2008$} \\
\hline 10 & 13.030 & 34.911 & $3.4 \pm 0.19$ & $29 \pm 1.3$ & $41 \pm 1.2$ & $3.7 \pm 0.37$ & $0.71 \pm 0.037$ & $1.1 \pm 0.13$ \\
\hline 20 & 13.030 & 34.911 & $3.0 \pm 0.21$ & $31 \pm 1.1$ & $41 \pm 1.2$ & $2.9 \pm 0.29$ & $0.74 \pm 0.035$ & $0.95 \pm 0.11$ \\
\hline 50 & 13.043 & 34.913 & $3.5 \pm 0.20$ & $31 \pm 1.1$ & $41 \pm 1.2$ & $3.6 \pm 0.36$ & $0.76 \pm 0.035$ & $1.0 \pm 0.12$ \\
\hline 70 & 13.047 & 34.913 & $1.7 \pm 0.15$ & $30 \pm 1.0$ & $41 \pm 1.2$ & $2.9 \pm 0.29$ & $0.74 \pm 0.032$ & $1.7 \pm 0.23$ \\
\hline 100 & 12.404 & 34.837 & $4.1 \pm 0.23$ & $33 \pm 1.3$ & $41 \pm 1.2$ & $1.1 \pm 0.11$ & $0.80 \pm 0.041$ & $0.27 \pm 0.030$ \\
\hline \multicolumn{9}{|c|}{$\mathrm{C} 7,178^{\circ} 22^{\prime} \mathrm{E}, 42^{\circ} 45^{\prime} \mathrm{S}, 1124 \mathrm{~m}, 05-28-2008$} \\
\hline 10 & 14.619 & 35.209 & $3.8 \pm 0.21$ & $31 \pm 1.1$ & $42 \pm 1.3$ & $3.3 \pm 0.33$ & $0.76 \pm 0.036$ & $0.87 \pm 0.099$ \\
\hline 25 & 14.611 & 35.208 & $3.5 \pm 0.22$ & $31 \pm 1.1$ & $42 \pm 1.3$ & $2.9 \pm 0.29$ & $0.75 \pm 0.035$ & $0.82 \pm 0.096$ \\
\hline 35 & 14.570 & 35.197 & $3.6 \pm 0.19$ & $31 \pm 1.1$ & $42 \pm 1.3$ & $3.1 \pm 0.31$ & $0.71 \pm 0.036$ & $0.86 \pm 0.098$ \\
\hline 50 & 14.034 & 35.083 & $3.4 \pm 0.19$ & $29 \pm 1.1$ & $41 \pm 1.2$ & $2.9 \pm 0.29$ & $0.70 \pm 0.033$ & $0.85 \pm 0.097$ \\
\hline 75 & 13.549 & 34.971 & $2.8 \pm 0.21$ & $31 \pm 1.1$ & $41 \pm 1.2$ & $3.4 \pm 0.34$ & $0.75 \pm 0.036$ & $1.2 \pm 0.15$ \\
\hline 120 & 13.139 & 35.052 & $5.5 \pm 0.23$ & $35 \pm 1.3$ & $41 \pm 1.2$ & $1.9 \pm 0.19$ & $0.85 \pm 0.030$ & $0.34 \pm 0.037$ \\
\hline 250 & 10.089 & 34.705 & $4.0 \pm 0.27$ & $35 \pm 1.3$ & $41 \pm 1.2$ & $1.6 \pm 0.16$ & $0.85 \pm 0.030$ & $0.39 \pm 0.047$ \\
\hline 500 & 8.409 & 34.563 & $4.8 \pm 0.22$ & $33 \pm 1.2$ & $41 \pm 1.2$ & $2.1 \pm 0.21$ & $0.81 \pm 0.030$ & $0.44 \pm 0.049$ \\
\hline 750 & 6.764 & 34.457 & $2.7 \pm 0.19$ & $40 \pm 1.4$ & $41 \pm 1.2$ & $0.76 \pm 0.076$ & $0.99 \pm 0.030$ & $0.28 \pm 0.035$ \\
\hline 1090 & 5.123 & 34.436 & $3.7 \pm 0.21$ & $36 \pm 1.2$ & $41 \pm 1.2$ & $1.6 \pm 0.16$ & $0.89 \pm 0.030$ & $0.42 \pm 0.049$ \\
\hline
\end{tabular}

C8, $178^{\circ} 49^{\prime}$ E, $42^{\circ} 49^{\prime}$ S, 1096 m, 05-29-2008

\begin{tabular}{|c|c|c|c|c|c|c|c|}
\hline 14.266 & 35.115 & $3.8 \pm 0.20$ & $30 \pm 1.1$ & $41 \pm 1.2$ & $2.3 \pm 0.23$ & $0.73 \pm 0.034$ & $0.62 \pm 0.071$ \\
\hline 14.231 & 35.115 & $3.5 \pm 0.22$ & $31 \pm 0.72$ & $41 \pm 1.2$ & $2.4 \pm 0.24$ & $0.75 \pm 0.028$ & $0.68 \pm 0.081$ \\
\hline
\end{tabular}


Table 1. Continued.

\begin{tabular}{|c|c|c|c|c|c|c|c|c|}
\hline $\begin{array}{r}\text { Depth } \\
\mathrm{m}\end{array}$ & $\begin{array}{r}\text { Temp. } \\
{ }^{\circ} \mathrm{C}\end{array}$ & Salinity & $\begin{array}{r}\text { Particulate }{ }^{234} \mathrm{Th} \\
\mathrm{mBq}^{-1}\end{array}$ & $\begin{array}{r}\text { Total }{ }^{234} \mathrm{Th} \\
\mathrm{mBq} 1^{-1}\end{array}$ & $\begin{array}{r}{ }^{238} \mathrm{U} \\
\mathrm{mBq}^{-1}\end{array}$ & $\begin{array}{r}\mathrm{POC} \\
\mu \mathrm{mol} \mathrm{C}^{-1}\end{array}$ & ${ }^{234} \mathrm{Th}:{ }^{238} \mathrm{U}$ & $\begin{array}{r}\mathrm{C}: \text { Th Ratio } \\
\mathrm{mmol} \mathrm{CBq}^{-1}\end{array}$ \\
\hline 50 & 220 & & \pm 0.21 & $4 \pm 1.3$ & $41 \pm 1.2$ & $2.2 \pm 0.22$ & $0.81\rfloor$ & $0.55=$ \\
\hline 75 & 14.214 & 35.104 & $4.0 \pm 0.20$ & $30 \pm 1.2$ & $41 \pm 1.2$ & $2.3 \pm 0.23$ & $0.73 \pm 0.036$ & $0.57 \pm 0.063$ \\
\hline 120 & 12.534 & 34.848 & $4.4 \pm 0.24$ & $34 \pm 1.3$ & $41 \pm 1.2$ & $1.2 \pm 0.12$ & $0.82 \pm 0.039$ & $0.28 \pm 0.032$ \\
\hline
\end{tabular}

C9, $174^{\circ} 36^{\prime} \mathrm{E}, 42^{\circ} 53^{\prime} \mathrm{S}, 1010 \mathrm{~m}, 05-30-2008$

$\begin{array}{rrrrrrrrr}10 & 14.069 & 35.113 & 4.0 \pm 0.21 & 33 \pm 1.2 & 41 \pm 1.2 & 2.1 \pm 0.21 & 0.80 \pm 0.038 & 0.53 \pm 0.060 \\ 20 & 14.031 & 35.104 & 4.3 \pm 0.23 & 37 \pm 1.3 & 41 \pm 1.2 & 2.5 \pm 0.25 & 0.88 \pm 0.040 & 0.57 \pm 0.064 \\ 45 & 14.049 & 35.107 & 4.4 \pm 0.21 & 35 \pm 1.2 & 41 \pm 1.2 & 1.6 \pm 0.16 & 0.85 \pm 0.039 & 0.36 \pm 0.039 \\ 70 & 13.885 & 35.066 & 4.6 \pm 0.20 & 33 \pm 1.4 & 41 \pm 1.2 & 1.7 \pm 0.17 & 0.79 \pm 0.040 & 0.38 \pm 0.042 \\ 100 & 13.736 & 35.033 & 3.1 \pm 0.20 & 34 \pm 1.2 & 41 \pm 1.2 & 2.1 \pm 0.21 & 0.83 \pm 0.038 & 0.69 \pm 0.083\end{array}$

$\mathrm{C} 10,178^{\circ} 17^{\prime} \mathrm{E}, 42^{\circ} 50^{\prime} \mathrm{S}, 662 \mathrm{~m}, 05-31-2008$

$\begin{array}{rrrrrrrrr}10 & 14.443 & 35.246 & 2.7 \pm 0.18 & 36 \pm 1.3 & 42 \pm 1.3 & 2.0 \pm 0.20 & 0.87 \pm 0.041 & 0.71 \pm 0.085 \\ 20 & 14.432 & 35.245 & 3.2 \pm 0.20 & 34 \pm 1.2 & 42 \pm 1.3 & 2.0 \pm 0.20 & 0.83 \pm 0.038 & 0.62 \pm 0.073 \\ 45 & 14.434 & 35.242 & 3.1 \pm 0.19 & 35 \pm 1.3 & 42 \pm 1.3 & 1.9 \pm 0.19 & 0.83 \pm 0.039 & 0.63 \pm 0.074 \\ 70 & 14.433 & 35.242 & 3.2 \pm 0.18 & 35 \pm 1.2 & 42 \pm 1.3 & 2.5 \pm 0.25 & 0.85 \pm 0.039 & 0.78 \pm 0.089 \\ 100 & 14.399 & 35.233 & 3.8 \pm 0.21 & 37 \pm 1.2 & 42 \pm 1.3 & 1.3 \pm 0.13 & 0.88 \pm 0.039 & 0.35 \pm 0.041\end{array}$

$\mathrm{C} 11,178^{\circ} 42^{\prime} \mathrm{E}, 42^{\circ} 58^{\prime} \mathrm{S}, 528 \mathrm{~m}, 06-01-2008$

\begin{tabular}{|c|c|c|c|c|c|c|c|c|}
\hline 10 & 13.748 & 35.041 & $2.6 \pm 0.17$ & $33 \pm 1.1$ & $41 \pm 1.2$ & $3.3 \pm 0.33$ & $0.80 \pm 0.035$ & $1.3 \pm 0.15$ \\
\hline 20 & 13.724 & 35.035 & $2.5 \pm 0.19$ & $31 \pm 1.0$ & $41 \pm 1.2$ & $2.9 \pm 0.29$ & $0.75 \pm 0.033$ & $1.2 \pm 0.15$ \\
\hline 45 & 13.684 & 35.025 & $2.4 \pm 0.17$ & $33 \pm 0.76$ & $41 \pm 1.2$ & $2.3 \pm 0.23$ & $0.80 \pm 0.030$ & $0.94 \pm 0.12$ \\
\hline 70 & 13.646 & 35.026 & $2.5 \pm 0.17$ & $32 \pm 0.74$ & $41 \pm 1.2$ & $2.0 \pm 0.20$ & $0.78 \pm 0.030$ & $0.80 \pm 0.097$ \\
\hline 100 & 13.411 & 34.994 & $2.6 \pm 0.19$ & $41 \pm 1.3$ & $41 \pm 1.2$ & $1.9 \pm 0.19$ & $1.0 \pm 0.043$ & $0.73 \pm 0.091$ \\
\hline \multicolumn{9}{|c|}{$\mathrm{C} 12,177^{\circ} 30^{\prime} \mathrm{E}, 43^{\circ} 42^{\prime} \mathrm{S}, 342 \mathrm{~m}, 06-02-2008$} \\
\hline 10 & 13.362 & 35.053 & $1.8 \pm 0.16$ & $31 \pm 1.0$ & $41 \pm 1.2$ & $1.6 \pm 0.16$ & $0.75 \pm 0.033$ & $0.87 \pm 0.12$ \\
\hline 20 & 13.366 & 35.053 & $2.5 \pm 0.19$ & $33 \pm 0.97$ & $41 \pm 1.2$ & $1.5 \pm 0.15$ & $0.79 \pm 0.033$ & $0.61 \pm 0.076$ \\
\hline 45 & 13.365 & 35.053 & $2.2 \pm 0.17$ & $33 \pm 1.1$ & $41 \pm 1.2$ & $1.6 \pm 0.16$ & $0.80 \pm 0.035$ & $0.72 \pm 0.090$ \\
\hline 70 & 13.366 & 35.053 & $2.1 \pm 0.16$ & $31 \pm 1.0$ & $41 \pm 1.2$ & $1.6 \pm 0.16$ & $0.74 \pm 0.033$ & $0.76 \pm 0.097$ \\
\hline 100 & 13.079 & 35.000 & $3.0 \pm 0.20$ & $32 \pm 1.3$ & $41 \pm 1.2$ & $1.2 \pm 0.12$ & $0.76 \pm 0.039$ & $0.42 \pm 0.050$ \\
\hline \multicolumn{9}{|c|}{$\mathrm{C} 13,178^{\circ} 31^{\prime} \mathrm{E}, 43^{\circ} 42^{\prime} \mathrm{S}, 422 \mathrm{~m}, 06-02-2008$} \\
\hline 10 & 13.603 & 35.025 & $2.0 \pm 0.17$ & $34 \pm 1.2$ & $41 \pm 1.2$ & $1.9 \pm 0.19$ & $0.82 \pm 0.037$ & $0.91 \pm 0.12$ \\
\hline 20 & 13.602 & 35.025 & $1.8 \pm 0.19$ & $35 \pm 1.2$ & $41 \pm 1.2$ & $2.1 \pm 0.21$ & $0.85 \pm 0.038$ & $1.2 \pm 0.17$ \\
\hline 45 & 13.608 & 35.024 & $2.1 \pm 0.16$ & $34 \pm 1.1$ & $41 \pm 1.2$ & $2.6 \pm 0.26$ & $0.83 \pm 0.037$ & $1.2 \pm 0.15$ \\
\hline 70 & 13.658 & 35.045 & $2.1 \pm 0.16$ & $32 \pm 1.1$ & $41 \pm 1.2$ & $2.0 \pm 0.20$ & $0.77 \pm 0.035$ & $0.95 \pm 0.12$ \\
\hline 100 & 11.842 & 34.842 & $3.5 \pm 0.21$ & $39 \pm 1.2$ & $41 \pm 1.2$ & $0.98 \pm 0.098$ & $0.94 \pm 0.041$ & $0.28 \pm 0.033$ \\
\hline \multicolumn{9}{|c|}{$\mathrm{C} 21,175^{\circ} 20^{\prime} \mathrm{E}, 43^{\circ} 68^{\prime} \mathrm{S}, 356 \mathrm{~m}, 06-02-2008$} \\
\hline 10 & 12.437 & 34.854 & $3.1 \pm 0.53$ & $29 \pm 1.8$ & $41 \pm 1.2$ & $2.4 \pm 0.24$ & $0.69 \pm 0.049$ & $0.76 \pm 0.15$ \\
\hline 20 & 12.442 & 34.854 & $4.5 \pm 0.62$ & $31 \pm 2.1$ & $41 \pm 1.2$ & $2.7 \pm 0.27$ & $0.75 \pm 0.056$ & $0.61 \pm 0.11$ \\
\hline 50 & 12.437 & 34.853 & $2.5 \pm 0.51$ & $36 \pm 1.9$ & $41 \pm 1.2$ & $2.5 \pm 0.25$ & $0.87 \pm 0.052$ & $1.0 \pm 0.23$ \\
\hline 70 & 12.438 & 34.853 & $4.3 \pm 0.51$ & $33 \pm 1.9$ & $41 \pm 1.2$ & $2.3 \pm 0.23$ & $0.81 \pm 0.051$ & $0.54 \pm 0.083$ \\
\hline 100 & 12.419 & 34.850 & $4.5 \pm 0.62$ & $37 \pm 2.0$ & $41 \pm 1.2$ & $2.4 \pm 0.24$ & $0.89 \pm 0.055$ & $0.52 \pm 0.088$ \\
\hline \multicolumn{9}{|c|}{ Mid-Salinity Stations } \\
\hline \multicolumn{9}{|c|}{$\mathrm{C} 5,176^{\circ} 30^{\prime} \mathrm{E}, 43^{\circ} 39^{\prime} \mathrm{S}, 367 \mathrm{~m}, 05-27-2008$} \\
\hline 10 & 11.433 & 34.481 & $7.6 \pm 0.26$ & $33 \pm 1.2$ & $41 \pm 1.2$ & $5.2 \pm 0.52$ & $0.80 \pm 0.037$ & $0.68 \pm 0.072$ \\
\hline 20 & 11.433 & 34.480 & $9.3 \pm 0.29$ & $31 \pm 1.2$ & $41 \pm 1.2$ & $5.6 \pm 0.56$ & $0.76 \pm 0.037$ & $0.60 \pm 0.063$ \\
\hline 50 & 11.647 & 34.536 & $6.6 \pm 0.24$ & $33 \pm 1.2$ & $41 \pm 1.2$ & $4.1 \pm 0.41$ & $0.82 \pm 0.039$ & $0.63 \pm 0.067$ \\
\hline 70 & 11.547 & 34.616 & $5.5 \pm 0.23$ & $35 \pm 1.3$ & $41 \pm 1.2$ & $3.6 \pm 0.36$ & $0.86 \pm 0.041$ & $0.65 \pm 0.071$ \\
\hline 100 & 10.886 & 34.701 & $3.0 \pm 0.21$ & $35 \pm 1.3$ & $41 \pm 1.2$ & $1.2 \pm 0.12$ & $0.85 \pm 0.040$ & $0.39 \pm 0.048$ \\
\hline
\end{tabular}


Table 1. Continued.

\begin{tabular}{|c|c|c|c|c|c|c|c|c|}
\hline $\begin{array}{r}\text { Depth } \\
\mathrm{m}\end{array}$ & $\begin{array}{r}\text { Temp. } \\
{ }^{\circ} \mathrm{C}\end{array}$ & Salinity & $\begin{array}{r}\text { Particulate }{ }^{234} \mathrm{Th} \\
\mathrm{mBq}^{-1}\end{array}$ & $\begin{array}{r}\text { Total }{ }^{234} \mathrm{Th} \\
\mathrm{mBql}^{-1}\end{array}$ & $\begin{array}{r}{ }^{238} \mathrm{U} \\
\mathrm{mBq}^{-1}\end{array}$ & $\begin{array}{r}\text { POC } \\
\mu \mathrm{mol} \mathrm{C} 1^{-1}\end{array}$ & ${ }^{234} \mathrm{Th}:{ }^{238} \mathrm{U}$ & $\begin{array}{r}\mathrm{C}: \text { Th Ratio } \\
\mathrm{mmol} \mathrm{CBq}^{-1}\end{array}$ \\
\hline 150 & 10.467 & 34.727 & $3.8 \pm 0.21$ & $36 \pm 1.2$ & $41 \pm 1.2$ & $1.3 \pm 0.13$ & $0.87 \pm 0.039$ & $0.36 \pm 0.041$ \\
\hline 250 & 9.539 & 34.664 & $3.9 \pm 0.21$ & $33 \pm 1.2$ & $41 \pm 1.2$ & $0.96 \pm 0.096$ & $0.82 \pm 0.039$ & $0.24 \pm 0.028$ \\
\hline 340 & 8.930 & 34.606 & $5.8 \pm 0.23$ & $31 \pm 1.3$ & $41 \pm 1.2$ & $1.9 \pm 0.19$ & $0.75 \pm 0.039$ & $0.33 \pm 0.035$ \\
\hline \multicolumn{9}{|c|}{$\mathrm{C} 6,178^{\circ} 22^{\prime} \mathrm{E}, 43^{\circ} 02^{\prime} \mathrm{S}, 340 \mathrm{~m}, 05-28-2008$} \\
\hline 10 & 11.660 & 34.536 & $8.8 \pm 0.27$ & $27 \pm 0.92$ & $41 \pm 1.2$ & $6.1 \pm 0.61$ & $0.65 \pm 0.030$ & $0.69 \pm 0.073$ \\
\hline 20 & 11.664 & 34.536 & $9.1 \pm 0.29$ & $29 \pm 1.1$ & $41 \pm 1.2$ & $5.7 \pm 0.57$ & $0.72 \pm 0.034$ & $0.63 \pm 0.066$ \\
\hline 50 & 11.672 & 34.538 & $9.5 \pm 0.28$ & $30 \pm 1.1$ & $41 \pm 1.2$ & $6.1 \pm 0.61$ & $0.74 \pm 0.034$ & $0.64 \pm 0.067$ \\
\hline 70 & 11.679 & 34.544 & $9.1 \pm 0.27$ & $30 \pm 1.2$ & $41 \pm 1.2$ & $5.4 \pm 0.54$ & $0.73 \pm 0.037$ & $0.59 \pm 0.062$ \\
\hline 100 & 11.247 & 34.740 & $3.3 \pm 0.21$ & $33 \pm 1.1$ & $41 \pm 1.2$ & $1.8 \pm 0.18$ & $0.80 \pm 0.035$ & $0.54 \pm 0.064$ \\
\hline 150 & 10.601 & 34.728 & $3.1 \pm 0.20$ & $35 \pm 2.6$ & $41 \pm 1.2$ & $1.1 \pm 0.11$ & $0.86 \pm 0.068$ & $0.35 \pm 0.042$ \\
\hline 250 & 8.966 & 34.605 & $1.8 \pm 0.17$ & $35 \pm 1.3$ & $41 \pm 1.2$ & $0.40 \pm 0.040$ & $0.86 \pm 0.040$ & $0.22 \pm 0.031$ \\
\hline 315 & 8.452 & 34.546 & $5.4 \pm 0.24$ & $24 \pm 1.0$ & $41 \pm 1.2$ & $1.6 \pm 0.16$ & $0.60 \pm 0.030$ & $0.29 \pm 0.031$ \\
\hline \multicolumn{9}{|c|}{$\mathrm{C} 14,178^{\circ} 20^{\prime} \mathrm{E}, 44^{\circ} 14^{\prime} \mathrm{S}, 526 \mathrm{~m}, 06-03-2008$} \\
\hline 10 & 10.837 & 34.340 & $11 \pm 0.29$ & $29 \pm 1.2$ & $41 \pm 1.2$ & $3.6 \pm 0.36$ & $0.72 \pm 0.036$ & $0.32 \pm 0.033$ \\
\hline 20 & 10.858 & 34.344 & $11 \pm 0.30$ & $32 \pm 1.0$ & $41 \pm 1.2$ & $2.0 \pm 0.20$ & $0.79 \pm 0.034$ & $0.18 \pm 0.018$ \\
\hline 45 & 11.021 & 34.390 & $12 \pm 0.29$ & $33 \pm 1.1$ & $41 \pm 1.2$ & $3.7 \pm 0.37$ & $0.82 \pm 0.036$ & $0.32 \pm 0.033$ \\
\hline 70 & 11.099 & 34.438 & $11 \pm 0.28$ & $32 \pm 1.1$ & $41 \pm 1.2$ & $3.3 \pm 0.33$ & $0.79 \pm 0.036$ & $0.31 \pm 0.032$ \\
\hline 100 & 10.485 & 34.578 & $3.5 \pm 0.21$ & $37 \pm 1.1$ & $41 \pm 1.2$ & $1.1 \pm 0.11$ & $0.90 \pm 0.038$ & $0.32 \pm 0.037$ \\
\hline 150 & 10.485 & 34.590 & n.d. & $40 \pm 1.3$ & $41 \pm 1.2$ & $0.79 \pm 0.079$ & $0.98 \pm 0.044$ & n.d. \\
\hline \multicolumn{9}{|c|}{$\mathrm{C} 15,177^{\circ} 48^{\prime} \mathrm{E}, 44^{\circ} 24^{\prime} \mathrm{S}, 716 \mathrm{~m}, 06-03-2008$} \\
\hline 10 & 11.109 & 34.386 & $11 \pm 0.31$ & $32 \pm 1.2$ & $41 \pm 1.2$ & $4.7 \pm 0.47$ & $0.80 \pm 0.038$ & $0.41 \pm 0.042$ \\
\hline 20 & 11.096 & 34.383 & $12 \pm 0.32$ & $28 \pm 1.1$ & $41 \pm 1.2$ & $4.4 \pm 0.44$ & $0.69 \pm 0.033$ & $0.38 \pm 0.039$ \\
\hline 50 & 10.905 & 34.353 & $7.2 \pm 0.26$ & $33 \pm 1.2$ & $41 \pm 1.2$ & $2.3 \pm 0.23$ & $0.81 \pm 0.038$ & $0.32 \pm 0.034$ \\
\hline 70 & 11.029 & 34.416 & $6.8 \pm 0.24$ & $38 \pm 1.4$ & $41 \pm 1.2$ & $1.7 \pm 0.17$ & $0.93 \pm 0.045$ & $0.26 \pm 0.027$ \\
\hline 100 & 10.879 & 34.626 & $2.8 \pm 0.21$ & $39 \pm 1.3$ & $41 \pm 1.2$ & $0.88 \pm 0.088$ & $0.96 \pm 0.043$ & $0.31 \pm 0.038$ \\
\hline \multicolumn{9}{|c|}{$\mathrm{C} 16,178^{\circ} 28^{\prime} \mathrm{E}, 44^{\circ} 33^{\prime} \mathrm{S}, 1078 \mathrm{~m}, 06-04-2008$} \\
\hline 10 & 10.361 & 34.265 & $11 \pm 0.30$ & $29 \pm 1.1$ & $40 \pm 1.2$ & $4.1 \pm 0.41$ & $0.72 \pm 0.036$ & $0.37 \pm 0.038$ \\
\hline 20 & 10.380 & 34.270 & $11 \pm 0.31$ & $29 \pm 1.2$ & $40 \pm 1.2$ & $3.6 \pm 0.36$ & $0.72 \pm 0.036$ & $0.33 \pm 0.034$ \\
\hline 40 & 10.409 & 34.277 & $9.1 \pm 0.28$ & $31 \pm 1.1$ & $40 \pm 1.2$ & $3.7 \pm 0.37$ & $0.76 \pm 0.035$ & $0.40 \pm 0.042$ \\
\hline 65 & 10.824 & 34.465 & $7.7 \pm 0.25$ & $29 \pm 1.0$ & $41 \pm 1.2$ & $2.3 \pm 0.23$ & $0.71 \pm 0.033$ & $0.30 \pm 0.032$ \\
\hline 100 & 9.767 & 34.531 & $3.8 \pm 0.22$ & $40 \pm 1.3$ & $41 \pm 1.2$ & $1.3 \pm 0.13$ & $0.98 \pm 0.044$ & $0.33 \pm 0.039$ \\
\hline \multicolumn{9}{|c|}{$\mathrm{C} 20,178^{\circ} 22^{\prime} \mathrm{E}, 43^{\circ} 37^{\prime} \mathrm{S}, 364 \mathrm{~m}, 06-06-2008$} \\
\hline 10 & 11.234 & 34.579 & $5.9 \pm 0.61$ & $35 \pm 2.1$ & $41 \pm 1.2$ & $4.1 \pm 0.41$ & $0.86 \pm 0.058$ & $0.69 \pm 0.099$ \\
\hline 20 & 11.235 & 34.579 & $5.2 \pm 0.71$ & $31 \pm 2.1$ & $41 \pm 1.2$ & $4.7 \pm 0.47$ & $0.76 \pm 0.056$ & $0.89 \pm 0.15$ \\
\hline 50 & 11.224 & 34.579 & $8.4 \pm 0.63$ & $36 \pm 2.3$ & $41 \pm 1.2$ & $4.2 \pm 0.42$ & $0.89 \pm 0.061$ & $0.50 \pm 0.062$ \\
\hline 70 & 11.223 & 34.579 & $6.5 \pm 0.58$ & $34 \pm 2.8$ & $41 \pm 1.2$ & $4.0 \pm 0.40$ & $0.82 \pm 0.073$ & $0.61 \pm 0.081$ \\
\hline 120 & 11.137 & 34.641 & $5.8 \pm 0.68$ & $35 \pm 2.3$ & $41 \pm 1.2$ & $2.9 \pm 0.29$ & $0.86 \pm 0.061$ & $0.50 \pm 0.076$ \\
\hline 250 & 9.409 & 34.608 & $6.1 \pm 0.61$ & $42 \pm 2.4$ & $41 \pm 1.2$ & $0.92 \pm 0.092$ & $1.1 \pm 0.066$ & $0.15 \pm 0.021$ \\
\hline 350 & 8.890 & 34.585 & $5.9 \pm 0.66$ & $31 \pm 2.3$ & $41 \pm 1.2$ & $1.3 \pm 0.13$ & $0.76 \pm 0.062$ & $0.23 \pm 0.034$ \\
\hline \multicolumn{9}{|c|}{$\mathrm{C} 22,175^{\circ} 20^{\prime} \mathrm{E}, 43^{\circ} 99^{\prime} \mathrm{S}, 470 \mathrm{~m}, 06-10-2008$} \\
\hline 10 & 10.870 & 34.593 & $5.5 \pm 0.58$ & $35 \pm 2.0$ & $41 \pm 1.2$ & $4.1 \pm 0.41$ & $0.85 \pm 0.056$ & $0.75 \pm 0.11$ \\
\hline 20 & 10.880 & 34.595 & $4.9 \pm 0.64$ & $36 \pm 2.3$ & $41 \pm 1.2$ & $4.0 \pm 0.40$ & $0.89 \pm 0.063$ & $0.82 \pm 0.14$ \\
\hline 50 & 10.811 & 34.581 & $6.8 \pm 0.60$ & $36 \pm 2.1$ & $41 \pm 1.2$ & $4.6 \pm 0.46$ & $0.89 \pm 0.058$ & $0.67 \pm 0.090$ \\
\hline 70 & 10.732 & 34.567 & $6.2 \pm 0.54$ & $34 \pm 2.1$ & $41 \pm 1.2$ & $3.3 \pm 0.33$ & $0.84 \pm 0.057$ & $0.53 \pm 0.071$ \\
\hline 100 & 10.689 & 34.565 & $5.5 \pm 0.69$ & $33 \pm 1.9$ & $41 \pm 1.2$ & $3.2 \pm 0.32$ & $0.80 \pm 0.053$ & $0.57 \pm 0.091$ \\
\hline
\end{tabular}

$\mathrm{D} 1,174^{\circ} 65^{\prime} \mathrm{E}, 43^{\circ} 65^{\prime} \mathrm{S}, 510 \mathrm{~m}, 06-10-2008$

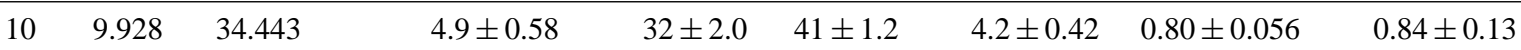


Table 1. Continued.

\begin{tabular}{|c|c|c|c|c|c|c|c|c|}
\hline $\begin{array}{r}\text { Depth } \\
\mathrm{m}\end{array}$ & $\begin{array}{r}\text { Temp. } \\
{ }^{\circ} \mathrm{C}\end{array}$ & Salinity & $\begin{array}{r}\text { Particulate }{ }^{234} \mathrm{Th} \\
\mathrm{mBq}^{-1}\end{array}$ & $\begin{array}{c}\text { Total }{ }^{234} \mathrm{Th} \\
\mathrm{mBq}^{-1}\end{array}$ & $\begin{array}{r}{ }^{238} \mathrm{U} \\
\mathrm{mBq}^{-1}\end{array}$ & $\begin{array}{r}\text { POC } \\
\mu \mathrm{mol} \mathrm{Cl}^{-1}\end{array}$ & ${ }^{234} \mathrm{Th}:{ }^{238} \mathrm{U}$ & $\begin{array}{r}\mathrm{C}: \text { Th Ratio } \\
\mathrm{mmol} \mathrm{CBq}^{-1}\end{array}$ \\
\hline 20 & 9.980 & 34.451 & $4.6 \pm 0.69$ & $32 \pm 2.3$ & $41 \pm 1.2$ & $4.3 \pm 0.43$ & $0.78 \pm 0.061$ & $0.95 \pm 0.17$ \\
\hline 50 & 10.005 & 34.455 & $4.8 \pm 0.58$ & $29 \pm 2.0$ & $41 \pm 1.2$ & $4.0 \pm 0.40$ & $0.71 \pm 0.053$ & $0.85 \pm 0.13$ \\
\hline 70 & 9.966 & 34.448 & $4.8 \pm 0.56$ & $33 \pm 2.1$ & $41 \pm 1.2$ & $4.1 \pm 0.41$ & $0.82 \pm 0.058$ & $0.86 \pm 0.13$ \\
\hline 100 & 9.945 & 34.452 & $4.6 \pm 0.64$ & $37 \pm 2.1$ & $41 \pm 1.2$ & $2.3 \pm 0.23$ & $0.91 \pm 0.058$ & $0.50 \pm 0.087$ \\
\hline \multicolumn{9}{|c|}{$\mathrm{C} 4-2^{\mathrm{b}}, 06-07-2008$} \\
\hline 10 & 10.975 & 34.542 & $7.9 \pm 0.73$ & $37 \pm 2.2$ & $41 \pm 1.2$ & $5.6 \pm 0.56$ & $0.90 \pm 0.059$ & $0.71 \pm 0.097$ \\
\hline 20 & 10.975 & 34.542 & $7.3 \pm 0.78$ & $39 \pm 2.5$ & $41 \pm 1.2$ & $5.3 \pm 0.53$ & $0.95 \pm 0.067$ & $0.72 \pm 0.11$ \\
\hline 50 & 10.979 & 34.542 & $8.1 \pm 0.66$ & $24 \pm 3.0$ & $41 \pm 1.2$ & $5.2 \pm 0.52$ & $0.59 \pm 0.075$ & $0.64 \pm 0.083$ \\
\hline 70 & 10.981 & 34.542 & $7.2 \pm 0.65$ & $31 \pm 2.2$ & $41 \pm 1.2$ & $5.2 \pm 0.52$ & $0.76 \pm 0.059$ & $0.73 \pm 0.099$ \\
\hline 100 & 10.980 & 34.543 & $8.5 \pm 0.79$ & $35 \pm 2.4$ & $41 \pm 1.2$ & $5.6 \pm 0.56$ & $0.85 \pm 0.065$ & $0.66 \pm 0.090$ \\
\hline \multicolumn{9}{|c|}{ Low Salinity Stations } \\
\hline \multicolumn{9}{|c|}{$\mathrm{C} 17,178^{\circ} 35^{\prime} \mathrm{E}, 44^{\circ} 21^{\prime} \mathrm{S}, 1186 \mathrm{~m}, 06-05-2008$} \\
\hline 10 & 9.699 & 34.206 & $7.8 \pm 0.26$ & $27 \pm 1.0$ & $40 \pm 1.2$ & $3.1 \pm 0.31$ & $0.66 \pm 0.032$ & $0.40 \pm 0.042$ \\
\hline 20 & 9.698 & 34.206 & $9.5 \pm 0.76$ & $30 \pm 1.1$ & $40 \pm 1.2$ & $2.4 \pm 0.24$ & $0.74 \pm 0.036$ & $0.26 \pm 0.033$ \\
\hline \multicolumn{9}{|c|}{$\mathrm{C} 22,175^{\circ} 20^{\prime} \mathrm{E}, 43^{\circ} 99^{\prime} \mathrm{S}, 470 \mathrm{~m}, 06-10-2008$} \\
\hline 45 & 9.704 & 34.206 & $7.5 \pm 0.25$ & $28 \pm 1.0$ & $40 \pm 1.2$ & $2.3 \pm 0.23$ & $0.68 \pm 0.033$ & $0.31 \pm 0.033$ \\
\hline 65 & 9.686 & 34.209 & $4.1 \pm 0.20$ & $32 \pm 1.1$ & $40 \pm 1.2$ & $1.5 \pm 0.15$ & $0.79 \pm 0.036$ & $0.35 \pm 0.039$ \\
\hline 100 & 9.363 & 34.383 & $3.5 \pm 0.22$ & $38 \pm 1.3$ & $41 \pm 1.2$ & $1.4 \pm 0.14$ & $0.94 \pm 0.043$ & $0.39 \pm 0.046$ \\
\hline 250 & 7.635 & 34.422 & $3.1 \pm 0.20$ & $39 \pm 1.3$ & $41 \pm 1.2$ & $1.0 \pm 0.10$ & $0.96 \pm 0.043$ & $0.33 \pm 0.040$ \\
\hline 500 & 6.843 & 34.370 & $3.7 \pm 0.21$ & $36 \pm 1.2$ & $41 \pm 1.2$ & $1.2 \pm 0.12$ & $0.89 \pm 0.041$ & $0.33 \pm 0.037$ \\
\hline 750 & 5.563 & 34.291 & $4.0 \pm 0.20$ & $36 \pm 1.2$ & $40 \pm 1.2$ & $0.61 \pm 0.061$ & $0.90 \pm 0.040$ & $0.15 \pm 0.017$ \\
\hline 1170 & 3.253 & 34.380 & $6.0 \pm 0.26$ & $31 \pm 1.1$ & $41 \pm 1.2$ & $1.0 \pm 0.10$ & $0.78 \pm 0.036$ & $0.17 \pm 0.019$ \\
\hline \multicolumn{9}{|c|}{$\mathrm{C} 18,177^{\circ} 43^{\prime} \mathrm{E}, 44^{\circ} 06^{\prime} \mathrm{S}, 942 \mathrm{~m}, 06-05-2008$} \\
\hline 10 & 9.699 & 34.237 & $7.2 \pm 0.26$ & $31 \pm 1.0$ & $40 \pm 1.2$ & $4.0 \pm 0.40$ & $0.77 \pm 0.034$ & $0.56 \pm 0.059$ \\
\hline 20 & 9.705 & 34.239 & $7.3 \pm 0.27$ & $31 \pm 1.0$ & $40 \pm 1.2$ & $3.9 \pm 0.39$ & $0.76 \pm 0.035$ & $0.54 \pm 0.057$ \\
\hline 45 & 9.715 & 34.241 & $7.7 \pm 0.26$ & $31 \pm 1.1$ & $40 \pm 1.2$ & $3.8 \pm 0.38$ & $0.76 \pm 0.035$ & $0.50 \pm 0.052$ \\
\hline 65 & 9.974 & 34.349 & $4.9 \pm 0.22$ & $36 \pm 1.2$ & $41 \pm 1.2$ & $2.8 \pm 0.28$ & $0.88 \pm 0.040$ & $0.57 \pm 0.062$ \\
\hline 100 & 9.768 & 34.464 & $2.8 \pm 0.21$ & $35 \pm 1.2$ & $41 \pm 1.2$ & $1.1 \pm 0.11$ & $0.86 \pm 0.039$ & $0.38 \pm 0.048$ \\
\hline 250 & 8.046 & 34.463 & $3.8 \pm 0.22$ & $38 \pm 1.3$ & $41 \pm 1.2$ & $0.90 \pm 0.090$ & $0.94 \pm 0.042$ & $0.24 \pm 0.028$ \\
\hline 500 & 7.013 & 34.384 & $2.6 \pm 0.19$ & $39 \pm 1.3$ & $41 \pm 1.2$ & $1.1 \pm 0.11$ & $0.96 \pm 0.043$ & $0.41 \pm 0.051$ \\
\hline 750 & 5.990 & 34.312 & $4.1 \pm 0.21$ & $37 \pm 1.2$ & $40 \pm 1.2$ & $0.74 \pm 0.074$ & $0.91 \pm 0.041$ & $0.18 \pm 0.020$ \\
\hline 890 & 5.553 & 34.290 & $4.7 \pm 0.40$ & $35 \pm 1.2$ & $40 \pm 1.2$ & $1.4 \pm 0.014$ & $0.87 \pm 0.040$ & $0.30 \pm 0.040$ \\
\hline \multicolumn{9}{|c|}{$\mathrm{C} 23,175^{\circ} 17^{\prime} \mathrm{E}, 44^{\circ} 23^{\prime} \mathrm{S}, 653 \mathrm{~m}, 06-08-2008$} \\
\hline 10 & 9.443 & 34.252 & $7.3 \pm 0.67$ & $35 \pm 2.2$ & $40 \pm 1.2$ & $3.7 \pm 0.37$ & $0.86 \pm 0.061$ & $0.50 \pm 0.069$ \\
\hline 20 & 9.436 & 34.250 & $5.3 \pm 0.74$ & $33 \pm 2.9$ & $40 \pm 1.2$ & $3.5 \pm 0.35$ & $0.81 \pm 0.076$ & $0.66 \pm 0.11$ \\
\hline 40 & 9.441 & 34.251 & $7.0 \pm 0.63$ & $33 \pm 2.9$ & $40 \pm 1.2$ & $3.4 \pm 0.34$ & $0.80 \pm 0.061$ & $0.48 \pm 0.064$ \\
\hline 60 & 9.497 & 34.270 & $5.4 \pm 0.60$ & $34 \pm 2.3$ & $40 \pm 1.2$ & $3.0 \pm 0.30$ & $0.84 \pm 0.061$ & $0.56 \pm 0.083$ \\
\hline 80 & 9.646 & 34.371 & $7.5 \pm 0.72$ & $32 \pm 2.2$ & $41 \pm 1.2$ & $3.0 \pm 0.30$ & $0.78 \pm 0.059$ & $0.40 \pm 0.055$ \\
\hline
\end{tabular}

a, b: second visits at Station C3 and C4.

\section{$3.4 \quad{ }^{234}$ Th export flux estimates}

The export flux of ${ }^{234} \mathrm{Th}$ at a specific depth horizon can be estimated by using the following equation:

$\frac{\delta A_{\mathrm{Th}}}{\delta t}=A_{\mathrm{U}} \lambda_{\mathrm{Th}}-A_{\mathrm{Th}} \lambda_{\mathrm{Th}}-P+V$ where $\frac{\delta A_{\text {Th }}}{\delta t}$ is the rate of change of total ${ }^{234} \mathrm{Th}$ activity, $A_{\mathrm{U}}$ is the ${ }^{238} \mathrm{U}$ activity estimated from the U-S relationship (Chen et al., 1986), $A_{\mathrm{Th}}$ is the total ${ }^{234} \mathrm{Th}$ activity, $\lambda_{\mathrm{Th}}$ is the decay constant for ${ }^{234} \mathrm{Th}\left(0.02876 \mathrm{~d}^{-1}\right), P$ is the net removal flux of ${ }^{234} \mathrm{Th}$ on particles, and $V$ is the sum of contributions from advection and diffusion. 


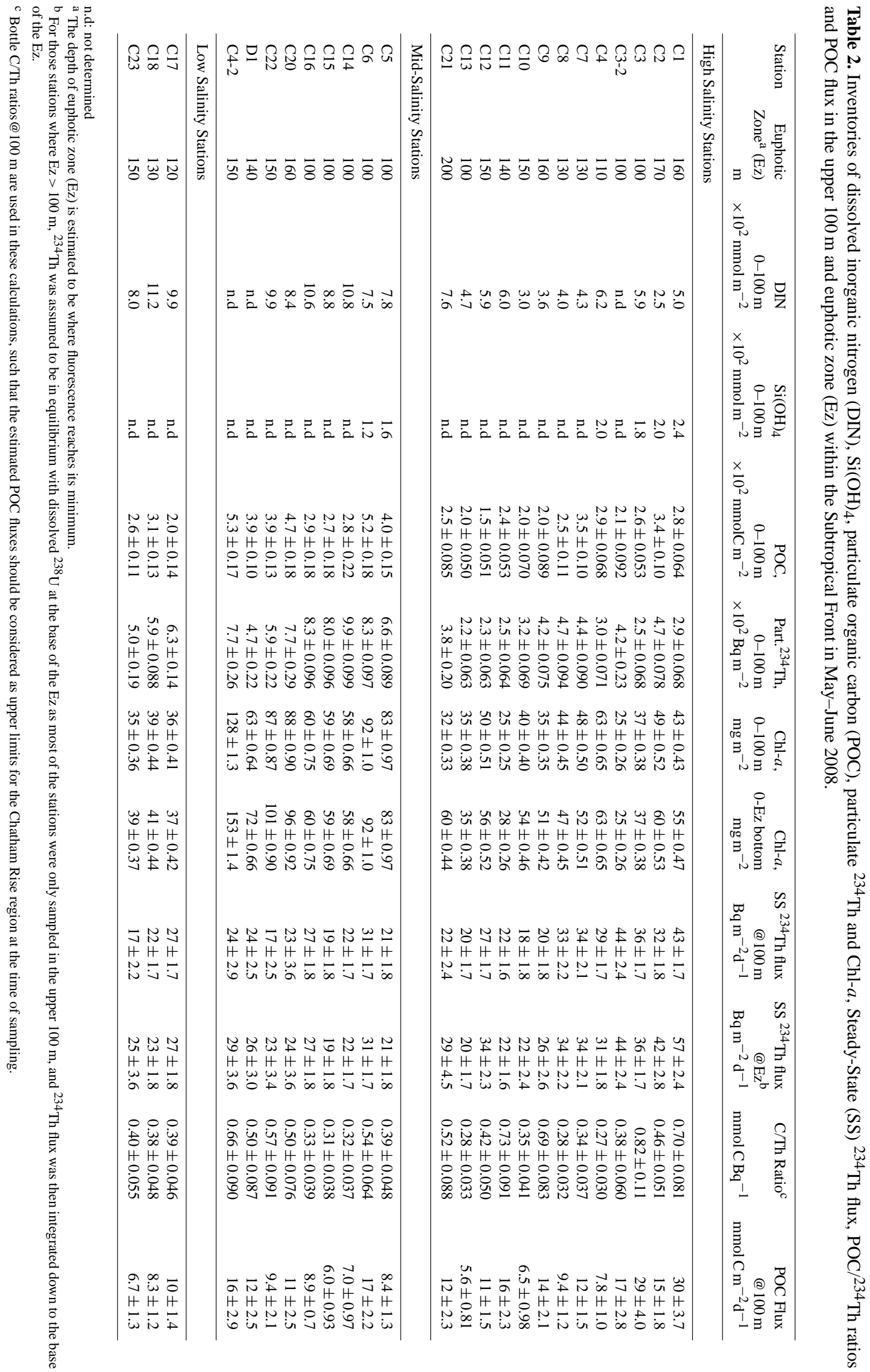



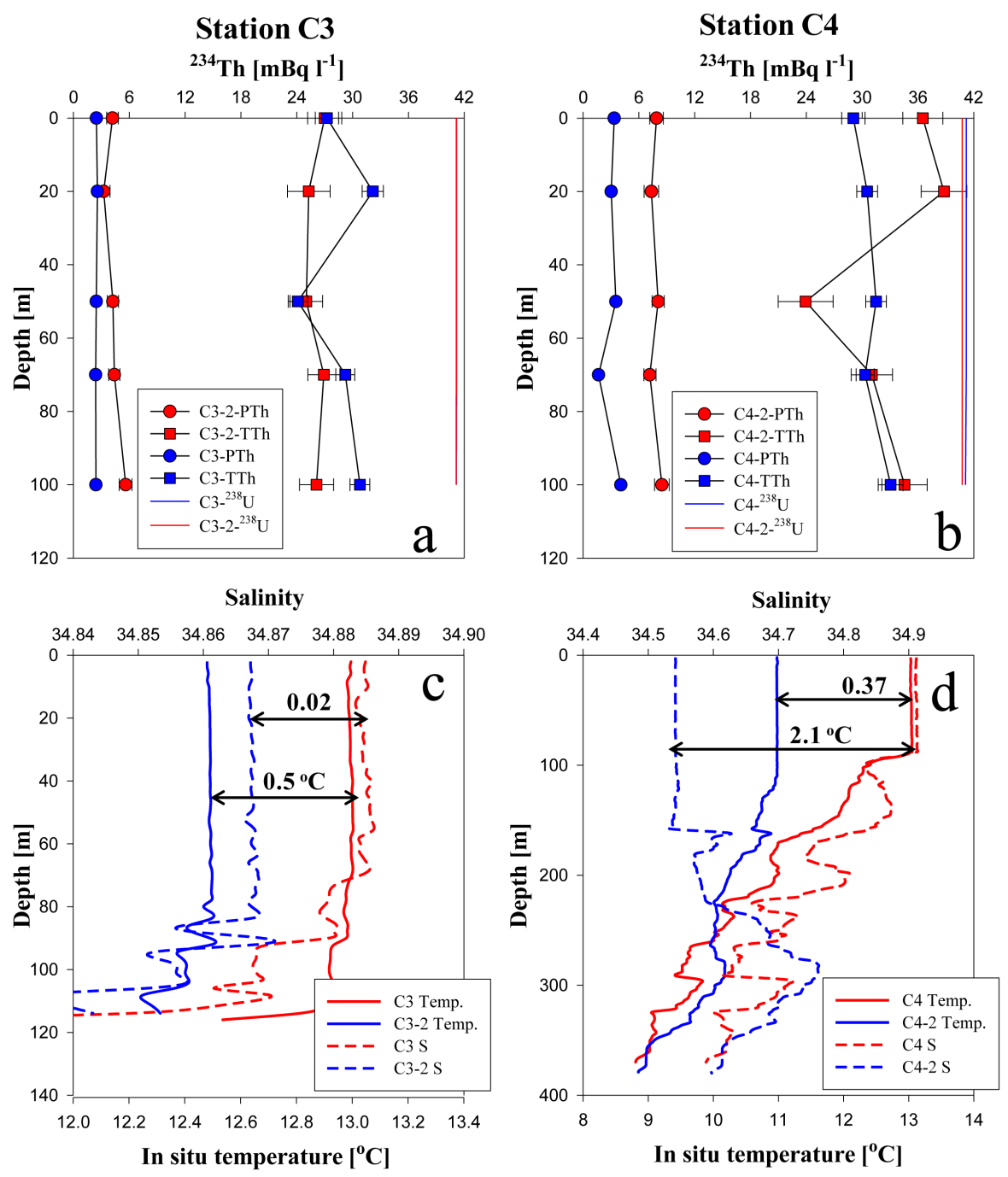

Fig. 7. (a) and (b) Vertical distributions of particulate and total ${ }^{234} \mathrm{Th}$ during the two visits to stations $\mathrm{C} 3$ and $\mathrm{C} 4$, with 16 days between C3 (26 May) and C3-2 (11 June) and 12 days between C4 (26 May) and C4-2 (7 June). (c) and (d) Vertical distributions of temperature and salinity at $\mathrm{C} 3$ and $\mathrm{C} 4$.

The Steady State (SS) model is applicable when little temporal change $\left(\frac{\delta A_{\mathrm{Th}}}{\delta t}\right)$ occurs in ${ }^{234} \mathrm{Th}$ activities or SS ${ }^{234} \mathrm{Th}$ flux is low (Savoye et al., 2006). However, when there are rapid changes in ${ }^{234} \mathrm{Th}$ activities, for example, during algal blooms or within physically dynamic regions, such as the STF, a non-steady state (NSS) ${ }^{234}$ Th flux model is generally necessary (Buesseler et al., 1992). To implement a NSS ${ }^{234}$ Th flux model, however, time-series observations from the same water mass are needed. In a practical sense, since there are difficulties in tracing specific water masses in the ocean, most studies have adopted a protocol to visit the same station at least twice during a particular study period (BenitezNelson et al., 2001b; Coppola et al., 2005; Kawakami and Honda, 2007). NSS ${ }^{234} \mathrm{Th}$ flux can then be calculated using the following equation (Buesseler et al., 1992):

$P_{\mathrm{NSS}}=\frac{\lambda\left[A_{\mathrm{U}}\left(1-e^{-\lambda t}\right)+A_{\mathrm{Th} 1} e^{-\lambda t}-A_{\mathrm{Th} 2}\right]}{1-e^{-\lambda t}}$

where $P_{\mathrm{NSS}}$ is the NSS ${ }^{234}$ Th export flux, and $A_{\mathrm{Th} 1}$ and $A_{\mathrm{Th} 2}$ are the ${ }^{234} \mathrm{Th}$ activities during the first and second visit, respectively. During our cruise, two stations (C3 and C4) were visited twice. The profiles of the temperature, salinity and ${ }^{234} \mathrm{Th}$ activity for these stations are shown in Fig. 7. At C3, differences in temperature and salinity of the upper $100 \mathrm{~m}$ between the two visits were $0.5^{\circ} \mathrm{C}$ and 0.02 , respectively. In contrast, at $\mathrm{C} 4$, larger differences were found: $2.1^{\circ} \mathrm{C}$ for temperature and 0.37 for salinity, which clearly indicated that different water masses were present at this location between the two visits. Due to the relatively minor changes in hydrography, however, we assume the same water mass was 


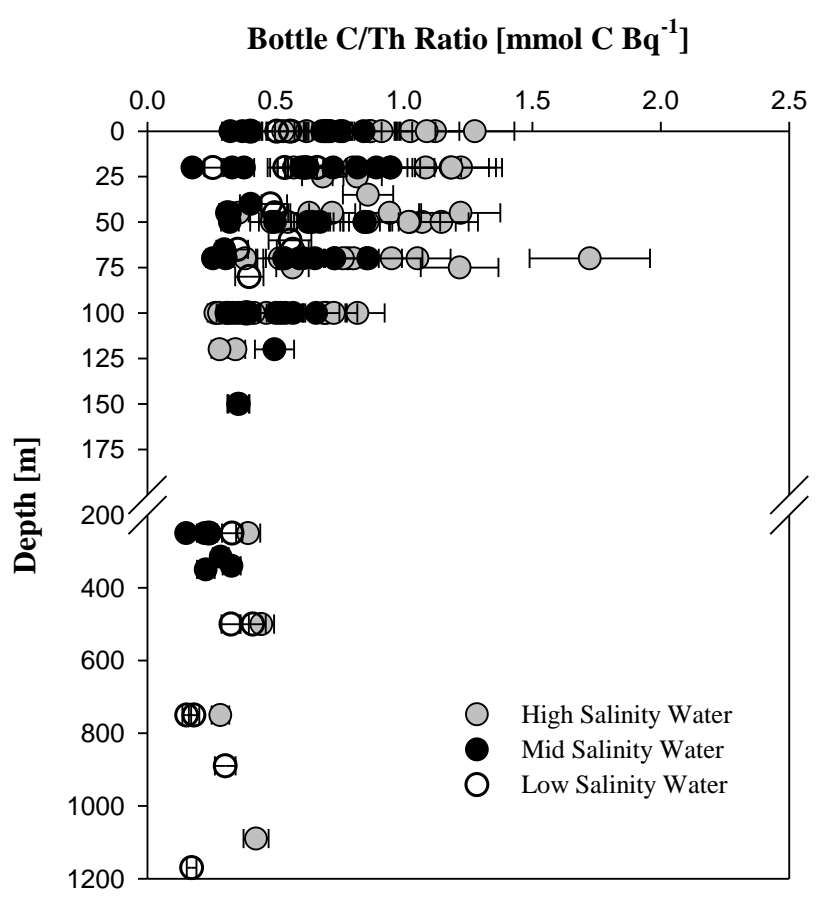

Fig. 8. Profiles of bottled POC/Th ratios for the three water types identified by their salinity differences (see Fig. 2).

sampled during the two visits to $\mathrm{C} 3$. Given the potential influence from the bottom re-suspension at this shallow station (see later), NSS ${ }^{234}$ Th flux from the upper $10 \mathrm{~m}$ was calculated as $4.1 \mathrm{~Bq} \mathrm{~m}^{-2} \mathrm{~d}^{-1}$. The $\mathrm{SS}^{234} \mathrm{Th}$ fluxes from the upper $10 \mathrm{~m}$ for the two visits to $\mathrm{C} 3$ were 4.0 and $4.1 \mathrm{~Bq} \mathrm{~m}^{-2} \mathrm{~d}^{-1}$, indicating that there was little temporal variability of ${ }^{234} \mathrm{Th}$ flux at this location. As such, the SS model is regarded to be mostly suitable for our ${ }^{234} \mathrm{Th}$ flux calculations for all the other locations sampled in the STF.

Physical processes may also influence the estimates of downward ${ }^{234}$ Th fluxes. Previous studies indicate that currents over the Chatham Rise can be strong, but variable, with alternating zones of convergence and divergence, although net zonal flows dominate along the northern and southern edges of Chatham Rise and predominantly meridional flows occur over the rise itself (Chiswell, 1996; Sutton, 2001). Given the little change observed in total ${ }^{234} \mathrm{Th}$ activities along the salinity gradients as shown in Fig. 6, however, we assumed that the horizontal contribution to the ${ }^{234} \mathrm{Th}$ fluxes was small compared to the downward vertical component of ${ }^{234} \mathrm{Th}$ export. Therefore, the $V$ term in Eq. (3) can be neglected in our ${ }^{234} \mathrm{Th}$ flux estimates.

Based on the above discussion, the $P$ term in Eq. (1) can then be solved as follows:

$P=\lambda_{\mathrm{Th}}\left(A_{\mathrm{U}}-A_{\mathrm{Th}}\right)$
In our case, the steady state (SS) downward flux of total ${ }^{234} \mathrm{Th}$ from the depth horizon of $100 \mathrm{~m}$ can be integrated by:

$P=\lambda_{\mathrm{Th}} \int_{0}^{100}\left(A_{\mathrm{U}}-A_{\mathrm{Th}}\right) d z$

The calculated ${ }^{234} \mathrm{Th}$ flux results for the upper $100 \mathrm{~m}$ of the water column are listed in Table $2 . \quad$ SS ${ }^{234} \mathrm{Th}$ fluxes range from $17 \pm 2.2 \mathrm{~Bq} \mathrm{~m}^{-2} \mathrm{~d}^{-1}$ to a maximum of $43 \pm 1.7 \mathrm{~Bq} \mathrm{~m}^{-2} \mathrm{~d}^{-1}$, with an average of $26 \pm 0.41 \mathrm{~Bq} \mathrm{~m}^{-2} \mathrm{~d}^{-1}(n=25) . \quad$ It is noted, however, that the highest value is from Station C3 with a bottom depth of $125 \mathrm{~m}$, and which consequently may be influenced by near-bottom re-suspension. As discussed previously, we also separated our ${ }^{234} \mathrm{Th}$ flux data into three groups. In low salinity waters, SS ${ }^{234} \mathrm{Th}$ fluxes varied from $17 \pm 2.2$ $\mathrm{Bq} \mathrm{m} \mathrm{m}^{-2} \mathrm{~d}^{-1}$ to $27 \pm 1.7 \mathrm{~Bq} \mathrm{~m}^{-2} \mathrm{~d}^{-1}$, with an average of $22 \pm 1.1 \mathrm{~Bq} \mathrm{~m}^{-2} \mathrm{~d}^{-1}(n=3)$. In mid-salinity waters, the flux varied from $17 \pm 2.5 \mathrm{~Bq} \mathrm{~m}^{-2} \mathrm{~d}^{-1}$ to $31 \pm 1.7 \mathrm{~Bq} \mathrm{~m}^{-2} \mathrm{~d}^{-1}$, with an average of $25 \pm 0.78 \mathrm{~Bq} \mathrm{~m}^{-2} \mathrm{~d}^{-1}(n=9)$. In high salinity waters, $\mathrm{SS}{ }^{234} \mathrm{Th}$ fluxes were similar to those in midsalinity waters, namely, $18 \pm 1.1 \mathrm{~Bq} \mathrm{~m}^{-2} \mathrm{~d}^{-1}$ to $44 \pm 2.4 \mathrm{~Bq}$ $\mathrm{m}^{-2} \mathrm{~d}^{-1}$, with an average of $29 \pm 0.53 \mathrm{~Bq} \mathrm{~m}^{-2} \mathrm{~d}^{-1}(n=13)$.

\subsection{Bottle $\mathrm{POC} /{ }^{234} \mathrm{Th}$ ratios}

Bottle $\mathrm{POC} / 234 \mathrm{Th}$ ratios are listed in Table 1. This ratio was quite variable, ranging from $0.15 \mathrm{mmolC} \mathrm{Bq}^{-1}$ to $1.7 \mathrm{mmol} \mathrm{C} \mathrm{Bq}^{-1}$. All POC/234 $\mathrm{Th}$ ratios were separated into three groups based on the previously defined salinity criteria, as shown in Fig. 8. Consistent with many prior studies, $\mathrm{POC} /{ }^{234} \mathrm{Th}$ was higher and more variable in the upper ocean, compared to the deep ocean (Buesseler et al., 2006). Interestingly, the ratio was generally lower in mid- and low salinity waters than in high salinity waters, reflecting different biological effects on carbon and thorium partitioning. This difference disappeared at and below $100 \mathrm{~m}$. At the export horizon of $100 \mathrm{~m}$, bottle $\mathrm{POC} /{ }^{234} \mathrm{Th}$ ratios varied from $0.27 \pm 0.030 \mathrm{mmol} \mathrm{CBq}^{-1}$ to $0.82 \pm 0.11 \mathrm{mmol} \mathrm{C} \mathrm{Bq}^{-1}$, with an average of $0.46 \pm 0.013 \mathrm{mmol} \mathrm{CBq}^{-1}$ (Table 1). Similar to ${ }^{234} \mathrm{Th}$ fluxes, no difference in $\mathrm{POC} /{ }^{234} \mathrm{Th}$ ratio was found among the three water types. In high salinity waters, the $\mathrm{POC} /{ }^{234} \mathrm{Th}$ ratio ranged from $0.27 \pm 0.030 \mathrm{mmol} \mathrm{CBq}^{-1}$ to $0.82 \pm 0.11 \mathrm{mmol} \mathrm{C} \mathrm{Bq}^{-1}$, with an average of $0.48 \pm 0.018 \mathrm{mmol} \mathrm{CBq}^{-1}$. In midsalinity waters, it varied from $0.31 \pm 0.038 \mathrm{mmol} \mathrm{CBq}^{-1}$ to $0.66 \pm 0.090 \mathrm{mmol} \mathrm{CBq}^{-1}$, with an average of $0.66 \pm 0.022 \mathrm{mmol} \mathrm{CBq}^{-1}$. In low salinity waters, $\mathrm{POC} /{ }^{234} \mathrm{Th}$ was less variable, ranging from $0.38 \pm 0.048 \mathrm{mmol} \mathrm{CBq}^{-1}$ to $0.40 \pm 0.055 \mathrm{mmol} \mathrm{C} \mathrm{Bq}^{-1}$, with an average of $0.39 \pm 0.029 \mathrm{mmol} \mathrm{C} \mathrm{Bq}^{-1}$. 


\section{Discussion}

\subsection{POC $/{ }^{234}$ Th ratios and POC export flux}

POC export fluxes can be estimated by multiplying ${ }^{234} \mathrm{Th}$ fluxes by the $\mathrm{POC} /{ }^{234} \mathrm{Th}$ ratios of sinking particles (Buesseler et al., 1992). To better constrain the POC export from the upper ocean using the ${ }^{234} \mathrm{Th}$ method, knowledge of the $\mathrm{POC} /{ }^{234} \mathrm{Th}$ ratios in sinking particles should be examined. However, in the present study, only bottle POC/ ${ }^{234} \mathrm{Th}$ data were collected during the cruise.

Many studies have shown that the bottle POC/ $/ 34$ Th ratio is typically higher than in pump and trap samples (Buesseler et al., 2006; Cai et al., 2008; Kawakami and Honda, 2007). Although bottle POC/ ${ }^{234} \mathrm{Th}$ ratios from particles in bottle samples are not expected to be particularly representative of sinking particles, it is reasonable to apply these measurements as an upper limit for the actual POC/ $/ 34$ Th ratio in sinking particles (Cai et al., 2008). A major advantage of this approach is that bottle filtrations enabled us to undertake high spatial resolution sampling of $\mathrm{POC} / 234 \mathrm{Th}$, compared to the spatially limited deployment of in situ pumps and sediment traps.

POC export fluxes estimated from SS ${ }^{234} \mathrm{Th}$ flux and bottle $\mathrm{POC} / 234 \mathrm{Th}$ ratios at the export depth horizon of $100 \mathrm{~m}$ are listed in Table 2. POC export ranged from $5.6 \pm 0.81$ to $30 \pm 3.7 \mathrm{mmol} \mathrm{C} \mathrm{m}^{-2} \mathrm{~d}^{-1}$, with an average of $12 \pm 0.41 \mathrm{mmol} \mathrm{C} \mathrm{m}^{-2} \mathrm{~d}^{-1}(n=25)$. As expected, POC export fluxes were similar among all three water types, which is in good agreement with the ${ }^{234} \mathrm{Th}$ flux distributions. POC flux varied from $5.6 \pm 0.81 \mathrm{mmol} \mathrm{C} \mathrm{m}^{-2} \mathrm{~d}^{-1}$ to $30 \pm 3.7 \mathrm{mmol} \mathrm{C} \mathrm{m}^{-2} \mathrm{~d}^{-1}$ in high salinity waters, with an average of $14 \pm 0.62 \mathrm{mmol} \mathrm{C} \mathrm{m}^{-2} \mathrm{~d}^{-1}$ $(n=13), \quad$ and from $6.7 \pm 1.3 \mathrm{mmol} \mathrm{C} \mathrm{m}^{-2} \mathrm{~d}^{-1}$ to $10 \pm 1.4 \mathrm{mmol} \mathrm{C} \mathrm{m}^{-2} \mathrm{~d}^{-1}$ in low salinity waters, with an average of $8.5 \pm 0.75 \mathrm{mmol} \mathrm{C} \mathrm{m}^{-2} \mathrm{~d}^{-1}(\mathrm{n}=3)$. In comparison, POC flux ranged from $6.0 \pm 0.93 \mathrm{mmol} \mathrm{C} \mathrm{m}^{-2} \mathrm{~d}^{-1}$ to $17 \pm 2.2 \mathrm{mmol} \mathrm{C} \mathrm{m}^{-2} \mathrm{~d}^{-1}$ in mid-salinity waters, with an average of $11 \pm 0.65 \mathrm{mmol} \mathrm{C} \mathrm{m}^{-2} \mathrm{~d}^{-1}(n=9)$.

Due to the lack of an effective methodology for determining downward POC export in such a physically dynamic area, few similar studies have been carried out in the STF over the Chatham Rise or even in other STF zones globally. Nodder (1997) attempted to test the hypothesis that the STF is a region of elevated export production in austral autumn via the deployment of a free-floating, surfacetethered, cylindrical sediment trap array on the northern side of the Chatham Rise. Mean POC fluxes were estimated to be $\sim 2.5 \mathrm{mmol} \mathrm{C} \mathrm{m}^{-2} \mathrm{~d}^{-1}$ at $200 \mathrm{~m}$ water depth, which is similar in magnitude to other locations in oligotrophic waters. Nodder and Alexander (1998) showed that spring particulate phosphorus fluxes at $\sim 100 \mathrm{~m}$ in the STF were almost double those in winter 1993. These earlier studies, however, were limited in their spatial coverage, which restricts the reliability of these results when extrapolated to the entire STF. Long-term moored sediment traps were deployed for a year at $300 \mathrm{~m}$ and $1000 \mathrm{~m}$ in the STF on the flanks of the Chatham Rise to investigate the seasonal variation of particle fluxes (Nodder and Northcote, 2001). POC export reached its maximum in spring $\left(14.8 \mathrm{mmol} \mathrm{C} \mathrm{m}^{-2} \mathrm{~d}^{-1}\right.$ at $300 \mathrm{~m}$ in the North and $4.8 \mathrm{mmol} \mathrm{C} \mathrm{m}^{-2} \mathrm{~d}^{-1}$ in the South), and annual average POC fluxes were calculated to be $10.1 \mathrm{mmol} \mathrm{C} \mathrm{m}^{-2} \mathrm{~d}^{-1}$ in the North, compared to $4.1 \mathrm{mmol} \mathrm{C} \mathrm{m}^{-2} \mathrm{~d}^{-1}$ in the South. POC export was also determined in frontal zones (including the STF and Polar Front) in the Indian Ocean sector of the Southern Ocean in summer using ${ }^{234} \mathrm{Th}$ methods (Coppola et al., 2005). At an export depth horizon of $100 \mathrm{~m}$, POC export varied from 0.1 to $2.5 \mathrm{mmol} \mathrm{C} \mathrm{m}^{-2} \mathrm{~d}^{-1}$, which is very low compared to other observations in the Southern Ocean (Buesseler et al., 2001b; Rutgers van der Loeff et al., 1997, 2002). The low diatom dominance in the water column was hypothesized to result in these lower than expected export fluxes. However, direct comparisons between these studies are difficult due to differences in the regional oceanography, observation duration, the chosen export depth horizon and applied methodologies. The export fluxes presented here for the STF on the Chatham Rise, are also only applicable to the season in which they were collected (i.e. late autumnearly winter), and it is expected that POC fluxes in the highly productive spring might be significantly enhanced in comparison (Nodder and Alexander, 1998; Nodder and Northcote, 2001). Nevertheless, the calculated POC fluxes using the ${ }^{234} \mathrm{Th}$ method are at least similar in magnitude to previous measurements of POC export in the same area, and provide us with the first appreciation of the substantial degree of spatial variability that could be expected in POC flux to the seafloor on the Chatham Rise. The POC flux estimated at $100 \mathrm{~m}$ in the present study decreased from west to east and from north to south across the rise (Fig. 9f). Benthic biomass and activity is generally higher on the southern flank of the Chatham Rise, compared to the crest and northern flank (Nodder et al., 2003, 2007; Probert and McKnight, 1993), which is somewhat incongruous with the lower POC fluxes suggested by this study and previous sediment trap results (Nodder and Northcote, 2001). Thus, it might be the quality, rather than the quantity, of POC supply to the benthos that has the most influence in structuring seafloor communities in this region (Nodder et al., 2003, 2007). 


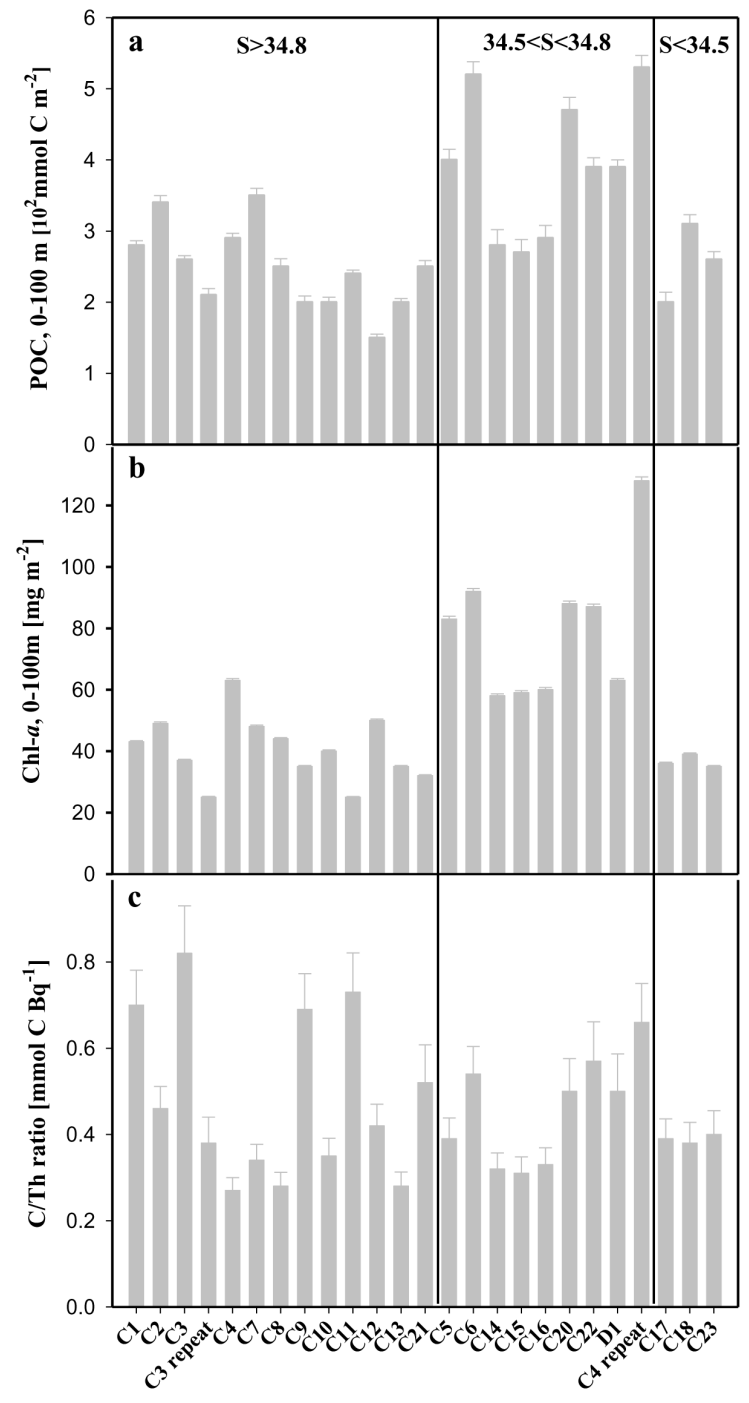

Stations

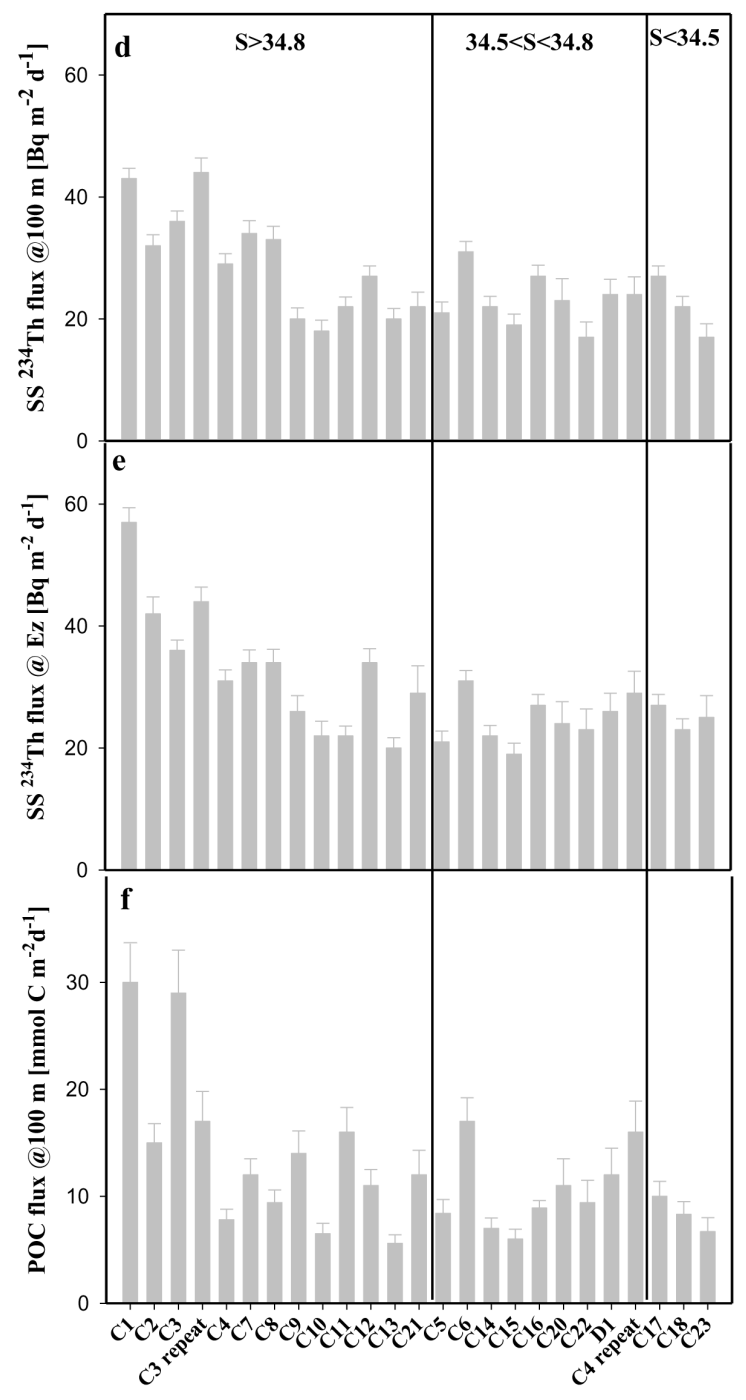

Stations

Fig. 9. Spatial distributions of: (a) POC inventory from $0-100 \mathrm{~m}$, (b) Chl- $a$ inventory from $0-100 \mathrm{~m}$, (c) $\mathrm{POC} / \mathrm{Th}$ ratio at $100 \mathrm{~m}$, (d) ${ }^{234} \mathrm{Th}$ fluxes at $100 \mathrm{~m}$, (e) ${ }^{234} \mathrm{Th}$ fluxes at the base of the Ez, and (f) POC fluxes at $100 \mathrm{~m}$. All stations are separated into three water types: low salinity $(\mathrm{S}<34.5)$, mid-salinity $(34.5<\mathrm{S}<34.8)$, and high salinity $(\mathrm{S}>34.8)$ waters.

Table 3. $P$ values derived from t-tests with unequal variance on inventories of POC, Particulate ${ }^{234} \mathrm{Th}$ and Chl- $a$, Steady-State (SS) ${ }^{234} \mathrm{Th}$ fluxes and POC fluxes at $100 \mathrm{~m}$ and euphotic zone (Ez) water depths for mid- vs. high salinity water and mid- vs. low salinity water.

\begin{tabular}{lrrr}
\hline Item & $\begin{array}{r}P \text { value } \\
\text { (Mid vs. High) }\end{array}$ & $\begin{array}{r}P \text { value } \\
(\text { Mid vs. Low })\end{array}$ & Significance \\
\hline POC, 0-100 m & 0.0021 & 0.020 & yes \\
Particulate Th, 0-100m & 0.000014 & 0.030 & yes \\
Chl- $a$, 0-100 m & 0.00031 & 0.00053 & yes \\
Chl- $a$, 0-Ez bottom & 0.0054 & 0.0018 & yes \\
SS 234Th flux@ 100m & 0.097 & 0.66 & no \\
SS 234Th flux@ Ez & 0.051 & 0.79 & no \\
POC flux@100 m & 0.10 & 0.22 & no \\
\hline
\end{tabular}




\subsection{Comparison between low, mid- and high salinity waters showing insignificant export production enhancement in the STF}

As shown in the Results section, Chl- $a$, POC and particulate ${ }^{234}$ Th data suggest enhanced biological particle production in the upper $100 \mathrm{~m}$ of mid-salinity waters, compared to low and high salinity waters within the STF. This trend continues to hold if we integrate these data from the sea surface to $100 \mathrm{~m}$ water depth (Table 2). For example, the average inventories of Chl- $a$, POC and particulate ${ }^{234} \mathrm{Th}$ activities of $80 \pm 0.30 \mathrm{mg} \mathrm{m}^{-2}, \quad(3.9 \pm 0.056) \times 10^{2} \mathrm{mmol} \mathrm{C} \mathrm{m}^{-2}$, and $(7.5 \pm 0.060) \times 10^{2} \mathrm{~Bq} \quad \mathrm{~m}^{-2}$, respectively, in mid-salinity waters, were high, compared to $40 \pm 0.30 \mathrm{mg} \mathrm{m}^{-2}, \quad(2.5 \pm 0.022) \times 10^{2} \mathrm{mmol} \mathrm{C} \mathrm{m}^{-2}$, and $(3.4 \pm 0.030) \times 10^{2} \mathrm{~Bq} \mathrm{~m}^{-2}$ in high salinity waters and $37 \pm 0.23 \mathrm{mg} \mathrm{m}^{-2},(2.6 \pm 0.073) \times 10^{2} \mathrm{mmol} \mathrm{C} \mathrm{m}^{-2}$, and $(5.7 \pm 0.084) \times 10^{2} \mathrm{~Bq} \mathrm{~m}^{-2}$ in low salinity waters. $T$-tests with unequal variance were carried out to compare the differences between the mid- and high salinity waters, and showed that $P$ values $(\alpha=0.05)$ for Chl- $a$, POC and particulate ${ }^{234} \mathrm{Th}$ were $0.0031,0.0021$, and 0.000014 , respectively (Table 3 ). $P$ values would be $0.00053,0.020$, and 0.030 , respectively for the same comparisons between mid- and low salinity waters. Such analyses indicate that the mid-salinity waters were statistically different from the other two water types. Note that the biological enhancement observed in the mid-salinity waters was also observed for primary production measured in a parallel study on the same cruise (Jill Schwarz, NIWA, personal communication). It is interesting that the enhancement of particulate ${ }^{234} \mathrm{Th}$ activity in mid-salinity waters was much stronger than for Chl- $a$ and POC.

The high Chl- $a$ biomass and/or PP levels in the STF have been well-defined in previous studies. For example, phytoplankton biomass in winter and spring were 4 and 6 times higher, respectively, in the STF than in adjacent low salinity SAW (Hall et al., 1999). The mean PP rate in winter could be as high as $22 \mathrm{mmol} \mathrm{C} \mathrm{m}^{-2} \mathrm{~d}^{-1}$, which was 4 times higher than that in the SAW (Bradford-Grieve et al., 1999). Remotesensing satellite data also show that the STF is characterized by year-round heightened pigment concentrations (Comiso et al., 1993; Murphy et al., 2001).

As described above, both DIN and $\mathrm{PO}_{4}$ were replete. In comparison, $\mathrm{Si}(\mathrm{OH})_{4}$ concentrations were in the range 0.39-3.4 $\mu_{\mathrm{mol}}{ }^{-1}$, which might indicate inhibition of diatom growth according to Chang and Gall (1998). Nevertheless, we tend to believe that $\mathrm{Si}(\mathrm{OH})_{4}$ was not yet limiting the PP because PP was significantly high at mid-salinity as compared to high salinity water regime despite the $\mathrm{Si}(\mathrm{OH})_{4}$ concentrations being similar at these sites.
Moreover, other studies have proposed that the elevated iron levels caused by mixing induced by the shallow bathymetry of Chatham Rise and/or the atmospheric deposition from Australian dust may lead to such high biomass/PP levels in the STF to the east of New Zealand, a process frequently termed "natural iron fertilization". Indeed, Boyd et al. (2004) determined the dissolved iron concentrations and potential iron sources in a transect across the STF. Dissolved iron concentrations in frontal surface waters reached the highest values of $0.8 \mathrm{nmol}^{-1}$ (above the stress level of $0.2 \mathrm{nmoll}^{-1}$ ) at about $43^{\circ} \mathrm{S}$, which is coincident geographically with the crest of the Chatham Rise. Boyd et al. (2004) further noted that iron concentrations dropped dramatically to less than $0.2 \mathrm{nmoll}^{-1}$ within 1 degree of latitude to the north of this location.

In contrast to the enhancement of PP within the STF, little difference in POC and ${ }^{234} \mathrm{Th}$ fluxes were found among the three water types identified in the present study. A $t$-test comparing the POC and ${ }^{234}$ Th fluxes at the $100 \mathrm{~m}$ export horizon between high and mid-salinity waters resulted in $P$ values $(\alpha=0.05)$ of 0.097 and 0.10 , respectively (Table 3$)$. The same parameters were compared between the mid- and low salinity waters, and similar observations were apparent (see Table 3). These relationships still held when all parameters were integrated to the bottom of the Ez (instead of to a fixed norminal depth such as $100 \mathrm{~m}$ ), which is not surprising given the fact that sinking particles originate mainly within the Ez and shallow re-mineralization has frequently been found just below this depth (e.g. Buesseler and Boyd, 2009). Thus, there was no difference in POC and ${ }^{234}$ Th fluxes at $100 \mathrm{~m}$ and/or the base of the Ez between the three water types.

The reasons why the elevated PP levels in the STF frontal zone, especially in our mid-salinity waters, did not lead to an increase in POC export are still unclear. Note that our study area is characterized by abundant diatom production in most seasons (Boyd et al., 1999; Bradford-Grieve et al., 1997), which should have driven high POC export fluxes as in many oceanic regimes (Michaels and Silver, 1988). Therefore we suggest that there are other factors limiting POC export here. Similar scenarios of this decoupling between PP and POC export were also observed in most of the artificial iron fertilization experiments (see reviews by Boyd et al., 2007), and the limited export response was attributed to the consequence of complex functioning of the planktonic community structure (Buesseler et al., 2004) and/or bacterial re-mineralization and grazing pressure (dominance of microzooplankton grazing over mesozooplankton) (Boyd and Newton, 1995). In the present study, the most plausible reason causing the little enhancement of the export flux would be related to microzooplankton grazing. Indeed, Hall et al. (1999) demonstrated that $>78 \%$ of daily PP can be grazed by microzooplankton in the STF in austral spring and winter. In contrast, mesozooplankton grazing is likely to be in the order of only 1-2\% of daily PP (Bradford-Grieve et al., 1998). Unlike mesozooplanton, the fecal pellets produced 
by microzooplankton are smaller and readily remineralized in the upper ocean and may not contribute significantly to export flux (Boyd and Newton, 1995; Michaels and Silver, 1988), as also suggested by other studies in the STF region (Nodder and Gall, 1998; Zeldis et al., 2002).

\section{Conclusions}

The present study applied a high resolution ${ }^{234} \mathrm{Th}$ sampling technique to define the magnitude and distribution of POC export in the STF region, which revealed with greater confidence that the POC export fluxes were on the order of $5.6 \pm 0.81$ to $30 \pm 3.7 \mathrm{mmol} \mathrm{C} \mathrm{m}^{-2} \mathrm{~d}^{-1}$, with an overall average of $12 \pm 0.41 \mathrm{mmol} \mathrm{C} \mathrm{m}^{-2} \mathrm{~d}^{-1}(n=25)$. There was little spatial variation among low, mid- and high salinity waters within the STF in austral autumn-winter, despite differences in biological particle production, as inferred from fluorescence/Chl- $a$ profiles. The present study, on the other hand, confirmed that the STF region is characterized by elevated PP, in particular, in the mid-salinity waters ( $34.5<\mathrm{S}<34.8$ ), presumably stimulated by so-called natural iron fertilization processes (Boyd et al., 1999, 2004; Pollard et al., 2009).

The present study, therefore, implies that natural iron fertilization does not necessarily lead to the enhancement of POC export in STF regions. It must be pointed out that, compared to other natural/ artificial iron experiments (Boyd et al., 2007), the present study was carried out in a different season (late autumn-early winter cf. summer) and latitude (43-44 ${ }^{\circ}$ cf. $\left.>50-60^{\circ}\right)$. Therefore, we anticipate that variations in temperature, latitude, season and oceanographic region will induce different ecosystem responses.

Acknowledgements. This study was funded by the Foundation of Science, Research and Technology (New Zealand) via the Coasts and Oceans Outcome-Based Investment (C01X0501). KZ was supported by a NIWA Capability Fund Visiting Scientist grant for him to participate in the cruise TAN0806. The preparation of the manuscript was supported by the National Natural Science Foundation of China through grant NSFC 40821063 to MHD. We thank the Captain, officers and crew of R/V Tangaroa for their assistance in sample collection during TAN0806, as well as other voyage participants, especially Steve George for operating the CTD. Thanks were also given to Karl Safi for supporting the macronutrient data. We finally thank Pinghe Cai and Ken Buesseler for their constructive comments on early drafts of this paper.

Edited by: C. Robinson

\section{References}

Bacon, M. P., Cochran, J. K., Hirschberg, D., Hammar, T. R., and Fleer, A. P.: Export flux of carbon at the equator during the EqPac time-series cruises estimated from ${ }^{234} \mathrm{Th}$ measurements, Deep Sea Res. II, 43, 1133-1153, 1996.
Behrenfeld, M. J. and Falkowski, P. G.: A consumer's guide to phytoplankton primary productivity models, Limnol. Oceanogr., 42, 1479-1491, 1997.

Benitez-Nelson, C. R., Buesseler, K. O., Karl, D. M., and Andrews, J.: A time-series study of particulate matter export in the North Pacific Subtropical Gyre based on ${ }^{234} \mathrm{Th}:{ }^{238} \mathrm{U}$ disequilibrium, Deep Sea Res. I, 48, 2595-2611, 2001a.

Benitez-Nelson, C. R., Buesseler, K. O., Rutgers van der Loeff, M.M., Andrews, J., Ball, L., Crossin, G., and Charette, M. A.: Testing a new small-volume technique for determining ${ }^{234} \mathrm{Th}$ in seawater, J. Radioanal. Nucl. Chem., 248, 795-799, 2001 b.

Boyd, P. and Newton, P.: Evidence of the potential influence of planktonic community structure on the interannual variablity of particulate organic carbon flux, Deep Sea Res. I, 42, 619-639, 1995.

Boyd, P., LaRoche, J., Gall, M., Frew, R., and McKay, R. M. L.: Role of iron, light, and silicate in controlling algal biomass in subantarctic waters SE of New Zealand, J. Geophys. Res., Oceans, 104, 13395-13408, 1999.

Boyd, P., McTainsh, G., Sherlock, V., Richardson, K., Nichol, S., Ellwood, S., and Frew, R.: Episodic enhancement of phytoplankton stocks in New Zealand subantarctic waters: Contribution of atmospheric and oceanic iron supply, Global Biogeochem. Cy., 18, GB1029, doi:1029/2002GB002020, 2004.

Boyd, P., Jickells, T. D., Law, C. S., Blain, S., Boyle, E. A., Buesseler, K. O., Coale, K. H., Cullen, J. J., de Baar, H. J. W., Follows, M., Harvey, M., Lancelot, C., Levasseur, M., Owens, N. P. J., Pollard, R., Rivkin, R. B., Sarmiento, J., Schoemann, V., Smetacek, V., Takeda, S., Tsuda, A., Turner, S., and Watson, A. J.: Mesoscale Iron Enrichment Experiments 1993-2005: Synthesis and Future Directions, Science, 315, 612-617, 2007.

Bradford-Grieve, J. M., Chang, F. H., Gall, M., Pickmere, S., and Richards, F.: Size-fractionated phytoplanton standing stocks and primary production during austral winter and spring 1993 in Subtropical Convergence region near New Zealand., N. Z. J. Mar. Freshwater Res., 31, 201-224, 1997.

Bradford-Grieve, J., Murdoch, R. C., James, M., Oliver, M., and McLeod, J.: Mesozooplankton biomass, composition, and potential grazing pressure on phytoplankton during austral winter and spring 1993 in the Subtropical Convergence region near New Zealand, Deep Sea Res. I, 45, 1709-1737, 1998.

Bradford-Grieve, J. M., Boyd, P. W., Chang, F. H., Chiswell, S., Hadfield, M., Hall, J. A., James, M. R., Nodder, S. D., and Shushkina, E. A.: Pelagic ecosystem structure and functioning in the Subtropical Front region east of New Zealand in austral winter and spring 1993, J. Plankton Res., 21, 405-428, 1999.

Buesseler, K. O., Bacon, M. P., Cochran, J. K., and Livingston, H. D.: Carbon and nitrogen export during the JGOFS North Atlantic bloom experiment estimated from ${ }^{234} \mathrm{Th}:{ }^{238} \mathrm{U}$ disequilibria, Deep Sea Res., 39, 1115-1137, 1992.

Buesseler, K. O, Ball, L., Andrews, J., Benitez-Nelson, C. R., Belastock, R., Chai, F., and Chao, Y.: Upper ocean export of particulate organic carbon in the Arabian Sea derived from thorium-234, Deep Sea Res. II, 45, 2461-2487, 1998.

Buesseler K. O., Ball, L., Andrews, J., Cochran, J. K., Hirschberg, D. J., Bacon, M. P., Fleer, A. P., and Brzezinski, M. A.: Upper ocean export of particulate organic carbon and biogenic silica in the Southern Ocean along $170^{\circ}$ W, Deep Sea Res. II, 48, 42754297, 2001a. 
Buesseler K. O., Benitez-Nelson, C. R., Rutgers van der Loeff, M.M., Andrews, J., Ball, L., Crossin, G., and Charette, M. A.: An intercomparison of small- and large-volume techniques for thorium-234 in seawater, Mar. Chem., 74, 15-28, $2001 \mathrm{~b}$.

Buesseler, K. O., Andrews, J. E., Pike, S., and Charette, M. A.: The effect of iron fertilization on carbon sequestration in the Southern ocean, Science, 304, 414-417, 2004.

Buesseler, K. O., Benitez-Nelson, C. R., Moran, S. B., Burd, A., Charette, M., Cochran, J. K., Coppola, L., Fisher, N. S., Fowler, S. W., Gardner, W. D., Guo, L. D., Gustafsson, Ö., Lamborg, C., Masque, P., Miquel, J. C., Passow, U., Santschi, P. H., Savoye, N., Stewart, G., and Trull, T.: An assessment of particulate organic carbon to thorium-234 ratios in the ocean and their impact on the application of ${ }^{234} \mathrm{Th}$ as a POC flux proxy, Mar. Chem., 100, 213-233, 2006.

Buesseler, K. O., Lamborg, C., Cai, P., Escoube, R., Johnson, R., Pike, S., Masque, P., McGillicuddy, D., and Verdeny, E.: Particle fluxes associated with mesoscale eddies in the Sargasso Sea, Deep Sea Res. II, 55, 1426-1444, 2008.

Buesseler, K. O. and Boyd, P. W.: Shedding light on processes that control particle export and flux attenuation in the twilight zone of the open ocean, Limnol. Oceanogr., 54, 1210-1232, 2009.

Buesseler, K. O., Pike, S., Maiti, K., Lamborg, C. H., Siegel, D. A., and Trull, T. W.: Thorium-234 as a tracer of spatial, temporal and vertical variability in particle flux in the North Pacific, Deep Sea Res. I, 56, 1143-1167, 2009.

Cai, P. H., Dai, M. H., Lv, D. W., and Chen, W. F.: An improvement in the small-volume technique for determining thorium-234 in seawater, Mar. Chem., 100, 282-288, 2006.

Cai, P. H., Chen, W. F., Dai, M. H., Wan, Z. W., Wang, D. X., Li, Q., Tang, T. T., and Lv, D. W.: A high-resolution study of particle export in the southern South China Sea based on ${ }^{234} \mathrm{Th}$ : ${ }^{238}$ U disequilibrium, J. Geophys. Res. Oceans, 113, C04019, doi:10.1029/2007JC004268, 2008.

Chang, F. H. and Gall, M.: Phytoplankton assemblages and photosynthetic pigments during winter and spring in the Subtropical Convergence region near New Zealand, N. Z. J. Mar. Freshwater Res., 32, 515-530, 1998.

Chen, J. H., Edwards, R. L., and Wasserburg, G. J.: ${ }^{238} \mathrm{U},{ }^{234} \mathrm{U}$ and ${ }^{232}$ Th in Seawater, Earth. Planet. Sci. Lett., 80, 241-251, 1986.

Chen, W. F.: On the Export Fluxes, Seasonality and Controls of Particulate Organic Carbon in the Northern South China Sea, PhD dissertation, Xiamen Univ. Xiamen, China, 2008 (in Chinese).

Chiswell, S. M.: Variability in sea surface temperature around New Zealand from AVHRR images, N. Z. J. Mar. Freshwater Res., 28, 179-192, 1994.

Chiswell, S. M.: Variability in the Southland Current, New Zealand, N. Z. J. Mar. Freshwater Res., 30, 1-17, 1996.

Clementson, L. A., Parslow, J. S., Griffiths, F. B., Lyne, V. D., Mackey, D. J., Harris, G. P., McKenzie, D. C., Bonham, P. I., Rathbone, C. A., and Rintoul, S.: Controls on phytoplankton production in the Australasian sector of the subtropical convergence, Deep Sea Res. I, 45, 1627-1661, 1998.

Coale, K. H. and Bruland, K. W.: ${ }^{234}$ Th: ${ }^{238} \mathrm{U}$ disequilibria within the California Current, Limnol. Oceanogr. 30, 22-33, 1985.

Coale, K. H. and Bruland, K. W.: Oceanic stratified Euphotic Zone as elucidated by ${ }^{234} \mathrm{Th}:{ }^{238} \mathrm{U}$ disequilibria, Limnol. Oceanogr. 32, 189-200, 1987.

Cochran, J. K. and Masque, P.: Short-lived U/Th Series radionu- clides in the ocean: tracers for scavenging rates, export fluxes and particle dynamics, Rev. Mineral. Geochem., 52, 461-492, 2003.

Comiso, J. C., Mcclain, C. R., Sullivan, C. W., Ryan, J. P., and Leonard, C. L.: Coastal Zone Color Scanner Pigment Concentrations in the Southern Ocean and Relationships to Geophysical Surface-Features, J. Geophys. Res. Oceans, 98, 2419-2451, 1993.

Coppola, L., Roy-Barman, M., Mulsow, S., Povinec, P., and Jeandel, C.: Low particulate organic carbon export in the frontal zone of the Southern Ocean (Indian sector) revealed by ${ }^{234} \mathrm{Th}$, Deep Sea Res. I, 52, 51-68, 2005.

Dai, M. H. and Benitez-Nelson, C. R.: Colloidal organic carbon and ${ }^{234}$ Th in the Gulf of Maine, Mar. Chem., 74, 181-196, 2001.

Froneman, P. W., McQuaid, C. D., and Laubscher, R. K.: Sizefractionated primary production studies in the vicinity of the Subtropical Front and an adjacent warm-core eddy south of Africa in austral winter, J. Plankton Res., 21, 2019-2035, 1999.

Gall, M., Hawes, I., and Boyd, P.: Predicting rates of primary production in the vicinity of the Subtropical Convergence east of New Zealand, N. Z. J. Mar. Freshwater Res., 33, 443-455, 1999.

Hall, J. A., James, M. R., and Bradford-Grieve, J. M.: Structure and dynamics of the pelagic microbial food web of the Subtropical Convergence region east of New Zealand, Aquat. Microb. Ecol., 20, 95-105, 1999.

Heath, R. A.: Oceanic Fronts around Southern New Zealand, Deep Sea Res., 28A, 547-560, 1981.

Heath, R. A.: A review of the physical oceanography of the seas around New Zealand-1982, N. Z. J. Mar. Freshwater Res., 19, 79-124, 1985.

Kara, A. B., Rochford, P. A., and Hurlburt, H. E.: An optimal definition for ocean mixed layer depth, J. Geophys. Res. Oceans, 105, 16803-16821, 2000.

Kawakami, H. and Honda, M. C.: Time-series observation of POC fluxes estimated from ${ }^{234} \mathrm{Th}$ in the northwestern North Pacific, Deep Sea Res. I, 54, 1070-1090, 2007.

Knap, A., Michaels, A., Close A., Ducklow, H., and Dickson, A.: Protocols for the Joint Global Ocean Flux Study (JGOFS) core measurements, JGOFS Report Nr. 19: vi-170 pp. (Reprint of the IOC Manuals and Guides No. 29, UNESCO 1994), 1996.

Longhurst, A.: Ecological Geography of the Sea, Academic Press, San Diego, Californiam 398 pp., 1998.

Michaels, A. F. and Silver, M. W.: Primary production, sinking fluxes and the microbial food web, Deep Sea Res., 35, 473-490, 1988.

Murphy, R. J., Pinkerton, M. H., Richardson, K. M., BradfordGrieve, J. M., and Boyd, P. W.: Phytoplankton distributions around New Zealand derived from SeaWiFS remotely-sensed ocean colour data, N. Z. J. Mar. Freshwater Res., 35, 343-362, 2001.

Nodder, S. D.: Short-term sediment trap fluxes from Chatham Rise, southwest Pacific Ocean, Limnol. Oceanogr., 42, 777-783, 1997.

Nodder, S. D. and Alexander, B. L.: Sources of variability in geographical and seasonal differences in particle fluxes from shortterm sediment trap deployments, east of New Zealand, Deep Sea Res. I, 45, 1739-1764, 1998a.

Nodder, S. D. and Gall, M.: Pigment fluxes from the Subtropical Convergence region, east of New Zealand: relationship to planktonic community structure, N. Z. J. Mar. Freshwater Res., 32, 
441-465, 1998b.

Nodder, S. D. and Northcote, L. C.: Episodic particulate fluxes at southern temperate mid-latitudes $\left(42-45^{\circ} \mathrm{S}\right)$ in the Subtropical Front region, east of New Zealand, Deep Sea Res. I, 48, 833864, 2001

Nodder, S. D., Pilditch, C. A., Probert, P. K., and Hall, J.: Variability in benthic biomass and activity beneath the Subtropical Front, Chatham Rise, SW Pacific Ocean, Deep Sea Res. I, 50, 959-985, 2003.

Nodder, S. D., Duineveld, G. C. A., Pilditch, C. A., Sutton, P., Probert, P. K., Lavaleye, M. S., Withbaard, R., Chang, F. H., Hall, J., and Richardson, K.: Focusing of phytodetritus deposition beneath a deep-ocean front, Chatham Rise, New Zealand, Limnol. Oceanogr., 52, 299-314, 2007.

Orsi, A. H., Whitworth III, T., and Nowlin Jr., W. D.: On the meridional extent and fronts of the Antarctic Circumpolar Current, Deep Sea Res. I, 42, 641-673, 1995.

Pollard, R. T., Salter, I., Sanders, R. J., Lucas, M. I., Moore, C. M., Mills, R. A., Statham, P. J., Allen, J. T., Baker, A. R., Bakker, D. C. E., Charette, M. A., Fielding, S., Fones, G. R., French, M., Hickman, A. E., Holland, R. J., Hughes, J. A., Jickells, T. D., Lampitt, R. S., Morris, P. J., Nédélec, F. H., Nielsdóttir, M., Planquette, H., Popova, E. E., Poulton, A. J., Read, J. F., Seeyave, S., Smith, T., Stinchcombe, M., Taylor, S., Thomalla, S., Venables, H. J., Williamson, R., and Zubkov, M. V.: Southern Ocean deep-water carbon export enhanced by natural iron fertilization, Nature, 457, 577-581, 2009.

Probert, P. K. and McKnight, D. G.: Biomass of bathyal macrobenthos in the region of the Subtropical Covergence, Chatham Rise, New Zealand, Deep Sea Res. I, 40, 1003-1007, 1993.
Redfield, A. C., Ketchum, B. H., and Richards, F. A.: The influence of organisms on the composition of sea-water, in: The Sea, 2, edited by: Hill, M. N., Interscience Publishers, John Wiley \& Sons, 26-77, 1963.

Rutgers van der Loeff, M. M., Friedrich, J., and Bathmann, U. V.: Carbon export during the Spring Bloom at the Antarctic Polar Front, determined with the natural tracer ${ }^{234} \mathrm{Th}$, Deep Sea Res. II, 44, 457-478, 1997.

Rutgers van der Loeff, M. M., Buesseler, K. O., Bathmann, U. V., Hense, I., and Andrews, J.: Comparison of carbon and opal export rates between summer and spring bloom periods in the region of the Antarctic Polar Front, SE Atlantic., Deep Sea Res. II, 49, 3849-3869, 2002.

Savoye, N., Benitez-Nelson, C. R., Burd, A. B., Cochran, J. K., Charette, M., Buesseler, K. O., Jackson, G. A., Roy-Barman, M., Schmidt, S., and Elskens, M.: ${ }^{234}$ Th sorption and export models in the water column: a review, Mar. Chem., 100, 234-249, 2006.

Sutton, P.: Detailed structure of the Subtropical Front over Chatham Rise, east of New Zealand, J. Geophys. Res., Oceans, 106, 31045-31056, 2001.

Uddstrom, M. J. and Oien, N. A.: On the use of high-resolution satellite data to describe the spatial and temporal variability of sea surface temperatures in the New Zealand region, J. Geophys. Res., 104, 20729-20751, 1999.

Waples, J. T., Benitez-Nelson, C. R., Savoye, N., Rutgers van der Loeff, M. M., Baskaran, M., and Gustafsson, Ö.: An introduction to the application and future use of ${ }^{234} \mathrm{Th}$ in aquatic systems, Mar. Chem., 100, 166-189, 2006.

Zeldis, J., James, M. R., Grieve, J., and Richards, L.: Omnivory by copepods in the New Zealand Subtropical Frontal Zone, J. Plankton Res., 24, 9-23, 2002. 